(C) 2019 IEEE. Personal use of this material is permitted. Permission from IEEE must be obtained for all other uses, in any current or future media, including reprinting/republishing this material for advertising or promotional purposes, creating new collective works, for resale or redistribution to servers or lists, or reuse of any copyrighted component of this work in other works.

Title: Generation of Homogeneous VHR Time Series by Nonparametric Regression of Multisensor Bitemporal Images

This paper appears in: IEEE Transactions on Geoscience and Remote Sensing

Date of Publication: 2019

Author(s): Yady Tatiana Solano-Correa, Francesca Bovolo and Lorenzo Bruzzone

Volume:

Page(s):

DOI: 10.1109/TGRS.2019.2914397 


\title{
Generation of Homogeneous VHR Time Series by Non-parametric Regression of Multisensor Bitemporal Images
}

\author{
Yady Tatiana Solano-Correa, Member, IEEE, Francesca Bovolo, Senior Member, IEEE, \\ and Lorenzo Bruzzone, Fellow, IEEE
}

\begin{abstract}
The availability of multitemporal images acquired by several very high geometrical resolution (VHR) optical sensors makes it possible to build VHR image Time Series (TS) of images acquired over the same geographical area with a temporal resolution better than the one achievable when considering a single VHR sensor. However, such TS include images showing different characteristics from the geometrical, radiometrical and spectral viewpoint. Thus, there is a need of methods for building homogeneous VHR optical TS when using multispectral multisensor images. By focusing on the spectral domain, we propose a method to transform a VHR image into the spectral domain of another image in the same multisensor TS, but acquired by a different sensor. To this end, a prediction-based approach relying on a non-parametric regression method is employed to mitigate sensor-dependent spectral differences. The impact of possible changes occurred on the ground is mitigated by training the prediction model on un-changed samples, only. Experimental results obtained on VHR optical multisensor images confirm the effectiveness of the proposed approach.
\end{abstract}

Index Terms - VHR Time Series, Radiometric Normalization, Multisensor Prediction, Non-parametric Regression, Change Detection, Remote Sensing.

\section{INTRODUCTION}

A generation of satellite sensors (e.g., IKONOS, QuickBird, GeoEye-1, WorldView-2) is available that enables to acquire multitemporal images with Very High spatial Resolution (VHR). Such sensors open to a large set of new applications in the field of Multi-Temporal (MT) analysis (e.g., Change Detection (CD)) that require spatial detail information. Nevertheless, when a single VHR optical sensor is considered, Time Series (TS) are likely to show a poor temporal resolution and to include images with nonhomogeneous acquisition conditions (e.g., lack of similar light conditions, different acquisition angle). This is mainly due to

Manuscript received July 12, 2018; revised December 18, 2018 and March 22, 2019; accepted April 19, 2019. This work was supported by the Project "MS-TS-Analysis of Multisensor VHR Image Time Series" with DigitalGlobe Foundation. (Corresponding author: Francesca Bovolo.)

Y. T. Solano-Correa and F. Bovolo are with the Fondazione Bruno Kessler, Center for Information and Communication Technology, 38123 Trento, Italy (e-mail: solano@ fbk.eu, bovolo@fbk.eu)

L. Bruzzone is with the Department of Information Engineering and Computer Science Trento, University of Trento, I-38123 Trento, Italy (e-mail: bruzzone@ing.unitn.it).

Color versions of one or more of the figures in this paper are available online at http://ieeexplore.ieee.org.

Digital Object Identifier 10.1109/TGRS.2019.2914397 the satellite revisit period, the possible competing orders of different users on the satellite pointing, the limited life of a satellite mission, and weather conditions [1]-[5]. In order to mitigate the abovementioned limitations and to have TS showing a (very) high resolution, in both space and time, multisensor optical acquisitions can be considered for TS construction. In this way, the probability of having frequent and good images over the same geographical area of interest increases. However, the use of multisensor multitemporal images shows several issues. The main one is related to images consistency. Lack of consistency in MT images applications impacts on the outcomes accuracy and reliability [4]. On the one side, images in multisensor TS are affected by differences induced by the acquisition conditions (e.g., atmospheric conditions and acquisition system). Some of the differences in atmospheric conditions (e.g., cloud cover), and acquisition system (e.g., view angle and seasonal effects) affect single-sensor multitemporal image as well [1]-[4]. On the other side, multisensor TS poses the big challenge of having system intrinsic differences due to the type of sensor. The above-mentioned issues are mainly related to differences in: i) the geometrical resolution; ii) the radiometric resolution; and iii) the spectral resolution, range and bandwidth of the sensors [1], [2], [6], [7]. In conclusion, among the various aspects of image pre-processing for $\mathrm{CD}$ when considering multisensor heterogeneous images, there are two outstanding requirements: i) geometric and, ii) spectral homogenization.

The issues related to geometric homogenization have been widely studied in the literature for low to VHR images and for multisensor images as well [1], [2], [8]-[13]. Whereas the huge variability arising in the spectral domain is less investigated. VHR available sensors may show significant differences in this domain since sensors are available acquiring images over different spectral ranges (e.g., IKONOS acquires bands in the range $445-853 \mathrm{~nm}$, while Pleiades acquires in the range 430-950nm [14]). In the case where similar spectral ranges are considered, the number of bands and/or their width may differ (e.g., QuickBird has 4 bands whereas WorldView-2 has 8 bands over the same range). Thus, the effective usage of multisensor TS poses the issue of how to perform multisensor data spectral homogenization. In the literature, two kinds of approaches have been proposed for addressing radiometric differences in single-sensor: i) Absolute Radiometric Normalization (ARN) [6] and; ii) 
Relative Radiometric Normalization (RRN) [7]. ARN refers to the use of physical parameters and makes it possible to relate the Digital Number (DN) with the radiance and reflectance at the Earth surface. A considerable amount of work has been carried out in the literature to design ARN methods and with focus on atmospheric corrections [15]-[20]. Other works showed the importance of using physical quantities for improving the CD accuracy [4], [21], [22]. Nevertheless, the use of ARN methods is not that common, even for singlesensor images, since their application requires knowledge of both the sensor spectral profile and atmospheric conditions at the time of acquisition. When data from two sensors $S_{1}$ and $S_{2}$ are considered, their spectral information is less comparable from the physical viewpoint with respect to data acquired from the same sensor. Thus, the use of ARN methods is mandatory before performing any further analysis [1], [2], [4]. RRN refers to transformations at pixel level for image-to-image adaptation. RRN methods use one image as a reference and adjust the radiometric properties of the other image to match the reference [6], [20], [23]. Thus, normalized images appear as being acquired with the reference image sensor and under similar atmospheric and illumination conditions [23]. However, RRN methods do not remove the differences introduced by atmospheric conditions in MT images, whereas ARN ones do.

A variety of RRN methods have been developed in the literature for single-sensor images. Hall et al [23] developed a radiometric rectification technique that corrects or rectifies images of the same area by using landscape elements with nearly constant reflectance over time. Others used similar procedures [19], [24], [25]. The drawback of these kind of approaches is that the landscape elements are selected by visual inspection, which could result in a subjective radiometric normalization. Thus, further methods perform the correction by scene-to-scene histogram normalization [7], [26], [27], scene-to-scene correction using dark and bright targets [23] or Pseudo Invariant Features (PIF) [28]. Most RRN methods assume that a linear relationship exists among times $t_{1}$ and $t_{2}$. Thus, the mathematical model describing standard RRN for MT images involves a linear regression [15]. Such regression models are built according to invariant samples or target points known as Radiometric Control Set Samples (RCSS). Burns and Joyce [29], and Singh [30] developed different techniques to select RCSS; however, those techniques still lead to low accuracy. Heo and Fitzhugh [31] suggested a method for obtaining the optimal linear equation with a given set of target points, but the results are biased by the subjective selection of RCSS. Methods for sample selection exist also in the literature of change detection based on classifiers [32], [33]. However, they do not account for the radiometric normalization of having radiometrically representative samples and seldom consider the complex nature of multisensor multitemporal images. Many other RRN methods, based on a linear relationship, can be found in the literature [15]-[17], [20], [34]-[37]. Most of them assume images with the same spectral and geometric characteristics (single-sensor), and acquired by medium or high spatial resolution sensors, devoting poor attention to multisensor and VHR optical images [6], [7], [35]. This linear relation influences the normalization results and is usually assumed because of simplification of modeling [38]. However, and as shown in Fig. 1, when multisensor VHR images are considered, the assumption of a linear model is seldom satisfied, leading to critical limitations. In fact, a non-linear non-parametric regression model would better adjust to the multisensor VHR problem. The problem remains how to automatically select the proper RCSS to derive the model itself. Therefore, development of new homogenization methods suitable for multisensor MT VHR images becomes of great relevance where VHR intrinsic characteristics are considered.

This paper presents a novel method for the generation of homogeneous VHR TS focused on the mitigation of intrinsic spectral induced differences. It is based on non-parametric regression and aims at generating consistent multisensor information showing a homogeneous spectral representation. The proposed method jointly exploits the capabilities of ARN and RRN approaches, by adapting them to the complexity of multisensor and VHR images. A first homogenization is carried on by transforming DN to physical values (ARN) and by transforming the multisensor images into a common spatial resolution, by means of state-of-the-art methods [1], [2], [4], [8], [10], [11], [13]. The second homogenization step is based on RRN approaches and performs a non-parametric regression (prediction) to derive a model that represents the relationship between $S_{1}$ and $S_{2}$. The model is derived by means of a machine learning algorithm, i.e., Artificial Neural Networks (ANN) or Support Vector Regression (SVR), and by introducing a novel approach for the RCSS selection. The selection of reliable RCSS is based on a novel CD-driven approach that is based on three steps: i) selection of invariant features (both spectral and textural), ii) selection of unchanged samples by relaxing the standard threshold in state-of-the-art methods; and iii) reduction of unchanged samples to avoid overfitting in the non-parametric regression step. This last step reduces the RCSS by: i) selecting the unchanged samples common to both spectral and textural invariant features; ii) preserving the statistical distribution of samples, as per original images; and iii) guaranteeing a homogenous spatial distribution over the considered geographical area. To demonstrate the effectiveness of the homogenization procedure, MT information is extracted, specifically by applying CD by means of Change Vector Analysis (CVA) [39]. Experiments were carried out on MT multisensor VHR image pairs.

The remainder of this paper is structured as follows. Section II presents an overview of the most common radiometric normalization methods for remote sensing images. Section III illustrates the proposed method for generation of homogeneous VHR TS by non-parametric regression of multisensor MT images. Sections IV and V explain in detail the steps of the proposed approach. Section VI presents the MT information extraction as an evaluation mean. Section VII introduces the multisensor datasets used in the experiments, 

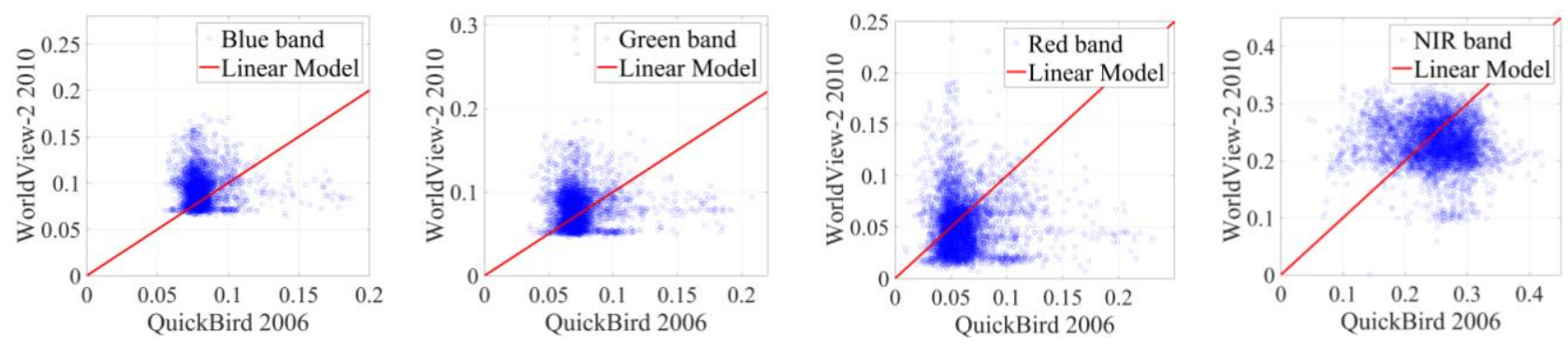

Fig. 1. Band by band scatterograms of QuickBird 2006 versus WorldView-2 2010 images in unchanged pixels.

describes the design of experiments and illustrates the experimental results. Finally, Section VIII draws the conclusions and illustrates future works.

\section{RADIOMETRIC NORMALIZATION METHODS FOR REMOTE SENSING IMAGES}

There are several reasons why a sensor response to a given target varies over the time. Among the more relevant ones, we have: i) changes in satellite sensor calibration, ii) differences in illumination and viewing angle, iii) variation in atmospheric effects, and iv) real changes on the ground [1], [3]. The goal of radiometric normalization methods is to remove or compensate for the above-mentioned effects, but the actual changes on the ground. In other words, the goal is to normalize the DN of images acquired under different conditions and map them to a common domain. Rather ARN or RRN methods are generally used in the literature to perform normalization [1], [2], [8]-[13], but they are seldom used jointly [1], [2], [38]. Since ARN methods have been widely standardized in the literature, in this section we focus the attention on RRN ones. RRN methods can be divided into three categories: i) statistical methods (e.g., standard deviation based method), ii) histogram matching methods and, iii) regression methods [7], [20]. Given the image-to-image relationship complexity, RRN methods used in the literature are mostly based on regression. RRN regression methods are based on 3 steps: i) selection of the model, ii) selection of RCSS and iii) estimation of normalization coefficients. The most commonly used model is linear because of its simplicity [38]. The assumption is that linear effects on data are stronger than nonlinear ones. Casseles and Garcia [19] showed that for low spatial resolution and single-sensor images, the relationship between the reference and subject image can be linear:

$$
Y_{m}=c_{m} X_{m}+d_{m}
$$

where $Y_{m}$ is the observed response in a given band of the reference image $Y, X_{m}$ is the corresponding vector of observed predictors of the subject image $X$ and, $c_{m}$ and $d_{m}$ are the normalization coefficients. Thus, in equation (1), the subject image $X$ is normalized by the reference image $Y$ by means of a linear regression. Nevertheless, the assumptions for applying a linear regression are seldom satisfied (especially if we consider multisensor VHR images) and the specific regression model is often unknown. A non-parametric regression analysis relaxes the assumptions of linearity, substituting it by a weaker assumption of a smooth population regression function of the form:

$$
Y_{m}=f\left(X_{m}\right)+\varepsilon_{m}
$$

where $f \in \mathcal{F}, \mathcal{F}$ is some class of regression functions, and $\varepsilon$ is the additive error with zero mean and constant variance. The main advantage here is that $\mathcal{F}$ is a rich enough class such as it is possible to approximate a very large set of regression functions. The cost of relaxing the linearity assumption implies higher computation burden, but with the gain of a more accurate estimate of the regression function. Examples of non-parametric regression methods are ANN [40] and SVR [41]. The second step is the selection of ideal RCSS. Ideal RCSS should [7], [42]: i) be preferably at the same elevation, ii) contain minimal amount of vegetation (when possible), iii) be invariant over the acquisition time, and iv) be distributed over the data spectrum such that the regression model can be reliable. Several methods have been introduced in the literature for the selection of RCSS [23], [28], [43]. In the next, some of the state of the art methods for selection of RCSS and further estimation of normalization coefficients, in low/medium resolution images, are briefly described together with their limits.

- Simple Regression (SR) [43]: Uses all pixels in both images to calculate normalization coefficients throughout least-squares. Because of this, it works well only when the considered images are stable over time, which is seldom satisfied in VHR images.

- Pseudo Invariant Features (PIF) [28]: Elements such as concrete, asphalt and rooftops are assumed statistically stable between the acquisition dates. Differences in the gray-level distribution of invariant objects are assumed to be linear and are corrected statistically to perform the normalization. It needs human intervention to extract the PIF set. Both linear assumption and human intervention are difficult while working with VHR images.

- Dark-Bright (DB) [23]: the average of a set of dark and bright pixels, extracted from the subject and reference image through Tasseled-Cap (TC) greenness-brightness transformation, is used to derive the normalization coefficients. Appropriate threshold values are required to obtain the dark and bright pixel sets. However, it is difficult to obtain them automatically. No TC coefficients are available for all VHR sensors.

- No Change set (NC) [43]: locates the statistical centers 
for stable land and stable water data clusters using the near-infrared (NIR) date 1 versus date 2 scatterograms to establish an initial regression line. At these wavelengths a distinct axis of "no-change" can be observed. Pixels falling within the $\mathrm{NC}$ region are used in the regression analysis of each band to compute normalization coefficients. Some limitations are: i) presence of both land and water areas is required and ii) two NIR bands are necessary. The latter issue is critical in VHR images, since often only one NIR channel is available.

- Iteratively Re-weighted Multivariate Alteration Detection (IR-MAD) [17]: uses MAD transformation to select nochange pixels in bi-temporal images by assuming a Gaussian distribution of the difference image. It is based on the linear combination of the $\mathrm{DN}$ of all the bands in the images, where the normalization coefficients are determined by applying standard Canonical Correlation Analysis (CCA). The final normalization is carried out by means of orthogonal linear regression. As it has already been pointed out, multisensor MT VHR images do not follow a linear relationship, neither the difference image does follow a Gaussian distribution [44].

If single sensor VHR MT images are considered, few of the above methods can be applied [17], [28]. For multisensor VHR images, none of the existing methods can be applied for normalization without modifications on: i) the regression model and/or ii) the selection of RCSS. This is because multisensor VHR image pairs may show stronger radiometric dissimilarities than single-sensor ones, even if change did not occur. This results in a lower number of spectrally invariant objects [11].

\section{Proposed Approach to UnSUPERVISED CD IN VHR MULTISPECTRAL IMAGES ACQUIRED BY DIFFERENT SENSORS}

In this section, the details of the propose approach to perform unsupervised CD in VHR multispectral multisensor images is presented. The approach generates homogeneous TS from multisensor MT VHR images by jointly exploiting the advantages of ARN and RRN methods. First, normalization of multisensor optical data by means of ARN and geometric normalization is applied. Second, RRN is conducted by means of the non-parametric regression of already absolutely corrected VHR multisensor images. The former step guarantees the multisensor comparison from both physical and geometrical view point [1], [2]. Whereas the latter guarantees the comparison from the spectral view point at pixel level. The method is based on non-parametric regression and uses the information provided by image at time $t_{2}$ acquired by sensor $S_{2}$ to effectively predict how spectral bands of the image acquired at $t_{1}$ by sensor $S_{1}$ would behave if acquired at $t_{2}$ by sensor $S_{1}$. Fig. 2 depicts the block scheme of the proposed method.

Let us consider two VHR optical images, acquired by sensors $S_{1}$ and $S_{2}$ over the same geographical area, at times $t_{1}$ and $t_{2}$ and with sizes $I_{1} \times J_{1}$ and $I_{2} \times J_{2}$, respectively. Let $a$ $(a=1,2, \ldots, A)$ and $b(b=1,2, \ldots, B)$ represent the generic multispectral bands of $S_{1}$ and $S_{2}$, respectively. Given the use of different sensors, the two images are likely to show different bandwidths, spatial resolutions and/or view angles. In other words, we assume that $S_{1}$ and $S_{2}$ may show: i) slightly different spatial resolution, ii) the same spectral range, iii) a different number of spectral bands $A$ and $B$, with $B>A$ (if $A>B$, the role of source and target images must be reversed), and iv) different spectral resolution. Thus, images acquired by the two sensors are similar, but not homogenous, and therefore not fully equivalent in the context of MT information extraction. Nevertheless, given the acquisition of images over the same spectral range, more than one band from $S_{2}$ acquires information over the same range of a single band from $S_{1}$. Because of this, more than one band from $S_{2}$ can be used to predict the corresponding bands of $S_{1}$ while deriving the homogenization model. The same process can be repeated over several pairs of images to homogeneous TS larger than two images.

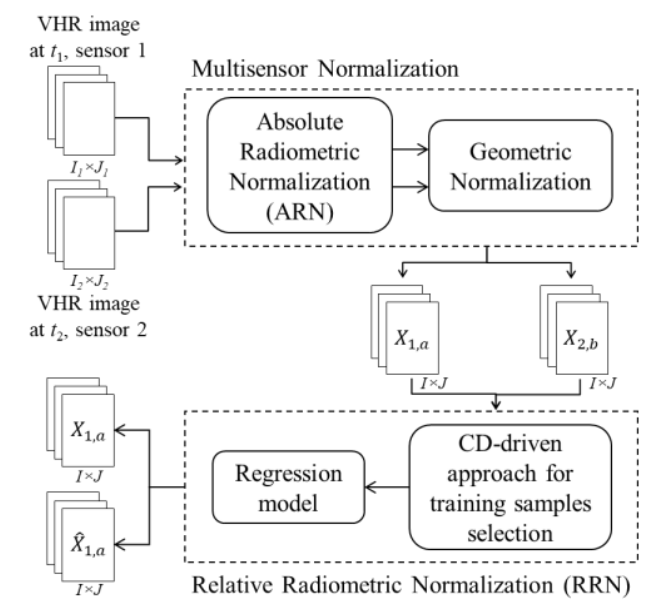

Fig. 2. Block scheme of the proposed method for generation of homogeneous VHR TS from multisensor MT images.

\section{Multisensor Time SerIEs HomogenizAtion}

A preliminary homogenization is carried on over the VHR multisensor images in order to mitigate differences induced by the use of multisensor acquisitions that result in both spectral and geometrical distortions [3]. In doing this, we guarantee a similarity, from the physical and geometrical viewpoints. To achieve this goal, two main steps are applied: i) ARN mitigation and ii) geometric normalization (see Fig. 2).

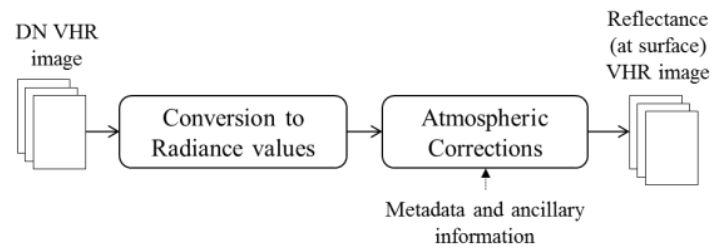

Fig. 3. Block scheme for ARN mitigation process.

ARN mitigation process is shown in Fig. 3. The original VHR images are first converted from DN to radiance values and then to Top Of Atmosphere (TOA) reflectance, known as at-surface reflectance. This is achieved by applying atmospheric corrections, resulting in images with the same physical meaning (further details can be found in [1], [2] and 
[4]). At the end of this step, some of the radiometrical differences among multisensor MT VHR images are corrected. Nevertheless, some differences remain that cannot be corrected or mitigated by ARN. Thus, a data-driven mechanism is later used to compensate for them (see sec. V).

Concerning geometrical differences, two sources can be identified: i) differences in the acquisition view angle, and ii) differences in the type of sensor because of multisensor acquisitions. The former can induce differences in the images when there are small changes in the topography and relief of the terrain. Whereas the latter results in differences in the spatial resolution and therefore in the correspondence of the same spatial position in the images. To achieve the geometric normalization, the block scheme shown in Fig. 4 is followed. Once again, this process does not fully correct for all the possible geometrical differences, but does help to mitigate for them. Surface reflectance images coming from both sensors are used as the input images for this step. They are orthorectified separately and then co-registered in order to guarantee a correspondence of each position on the ground in the MT images. The preliminary homogenized images are $X_{1, a}$ and $X_{2, b}$. Further details can be found in [1], [2].

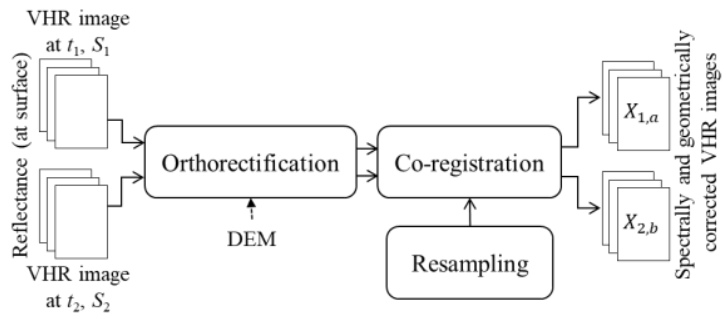

Fig. 4. Block scheme for the geometric normalization of multisensor VHR images.

\section{RELATIVE RADIOMETRIC NORMALIZATION}

With the preliminary homogenization step, we guarantee that $X_{1, a}$ and $X_{2, b}$ have common: i) spatial resolution, ii) image size $I \times J$, and iii) radiometric representation from the physical viewpoint. Yet, sensor induced differences in terms of radiometry and number of bands remain. To mitigate for these issues we perform further normalization by means of a novel RRN based on a non-parametric regression that guarantees a model suitable for multisensor VHR images. This model jointly accounts for the remaining issues due to the spectral, radiometrical and geometrical differences. To estimate the model, we need stable RCSS that ensure capturing the complex relationship between $S_{1}$ and $S_{2}$. Opposite to state-ofthe-art methods, RCSS are automatically selected. To this aim, a CD-driven approach is considered.

The block scheme for the proposed RRN approach is based on 4 steps (Fig. 5): i) selection of invariant features from $X_{1, a}$ and $X_{2, b}$; ii) selection of unchanged samples, iii) sub-sample of unchanged samples; and iv) non-parametric regression based on prediction and fusion with two phases, training and recalling. Most of these steps have been separately used in a different way in literature for single-sensor and low-resolution images.

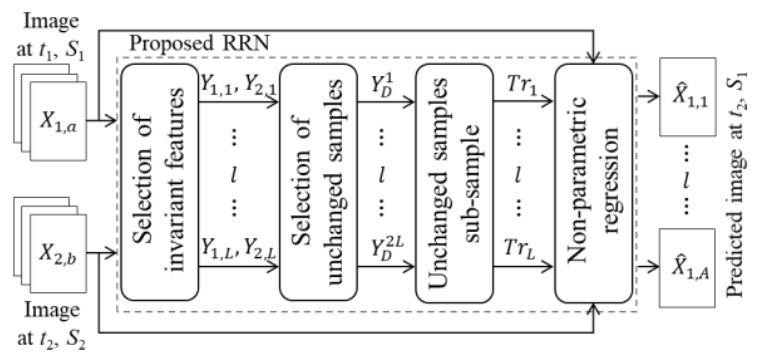

Fig. 5. Block scheme followed for the proposed RRN process.

\section{A. Selection of Invariant Features}

In order to properly model the radiometric relationship between $S_{1}$ and $S_{2}$, only un-changed (radiometrically invariant) samples are considered. To select them, spectral information is commonly used in literature. Nevertheless, the higher spatial details given by the use of VHR images, makes spectral information alone not enough to decide if a change has occurred or not. Spatial correlation should be used as well to decide if a pixel has indeed changed or not. In order to extract the spectral and spatial information, invariant spectral and spatial features are considered. The decision about which sample has changed or not can rely on a CD approach, where a pixel-by-pixel comparison is applied. Nevertheless, given that $X_{1, a}$ and $X_{2, b}$ show different spectral behaviors, $B>A$, the correspondence between the $a$ th and $b$ th bands is not one-toone. However, since $S_{1}$ and $S_{2}$ acquire images over the same spectral range, $L$ couples of bands $\left(X_{1, l}\right.$ and $\left.X_{2, l}\right)$ can be identified that show the most similar central wavelength and bandwidth, with $L \leq A<B$. From this set of most similar bands, a set of invariant features are derived for unchanged samples detection.

Even though atmospheric corrections have been applied, residual differences exist among spectral channels. This results in $L$ bands histogram shape difference and relative shift (see Fig. 6), even in case of no-change. Thus, compensation of spectral differences is carried out on the $L$ pairs in terms of shift. This pushes the peak values $\left(P_{\max _{S_{1}}}\right.$ and $\left.P_{\max _{S_{2}}}\right)$ to the same position in the spectral domain. The shift value is extracted as $P=\left|P_{\max _{S_{2}}}-P_{\max _{S_{1}}}\right|$. The set of spectral (spe) invariant features is then extracted from the histogram compensated bands $Y_{1, l, s p e}$ and $Y_{2, l, s p e}$, with $l \in[1, L]$.

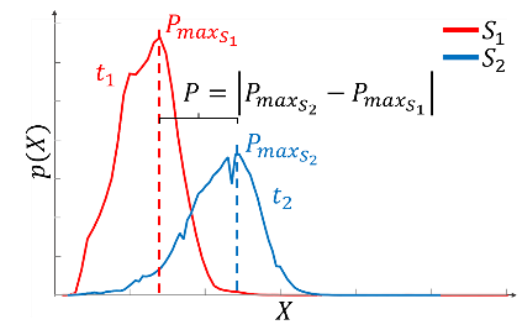

Fig. 6. Example of histogram shift of a pair of most similar bands in two different VHR sensors.

The high spatial variability of spectral signatures in VHR images, results in the increase of single date image interclass variability and thus in the variability of both change and no change classes. This phenomenon is enhanced by the temporal 
spectral variability induced by the use of multisensor VHR images. This results in a higher complexity in finding unchanged training samples. The effect can be mitigated by using other features that exploit the spatial details in VHR images. In the proposed method, textural features extracted from the $L$ most similar bands are employed as a complement to select the invariant training samples. We expect the spatial variability introduced by texture features in a single image to be temporally correlated. In other words, we expect the spatial variability to be stable in time where no changes occurred. Possible smoothing effects are prevented by the joint use of spectral and spatial features. A spatial invariant features set is defined by the textural $(t x t)$ features extracted from the $L$ most similar bands as $Y_{1, l, t x t}$ and $Y_{2, l, t x t}$, where $l \in[1, L]$.

\section{B. Unchanged Samples Selection}

In order to model the temporal and spectral relationship between $X_{1, a}$ and $X_{2, b}$, we select samples that are i) likely unchanged $\left(\omega_{n}\right)$ and ii) as much as possible representative of the spectral variability of unchanged information over the bands. The reliability of these samples is guaranteed by a CDdriven approach based on Univariate Image Difference (UID) and a conservative threshold. UID is applied to each pair of invariant features $\left(Y_{1, l}, Y_{2, l}\right)$ as:

$$
Y_{D}^{l}=\left|Y_{2, l}-Y_{1, l}\right|, l=1, \ldots, 2 L
$$

where $Y_{D}^{l}$ is the absolute difference image and $Y_{1, l}=$ $\left\{Y_{1, l, s p e}, Y_{1, l, t x t}\right\}$ and $Y_{2, l}=\left\{Y_{2, l, s p e}, Y_{2, l, t x t}\right\}$. The most reliable unchanged samples are selected as the ones showing a small difference value, i.e., below a certain threshold value $T^{l}(l=$ $1, \ldots, 2 L)$.

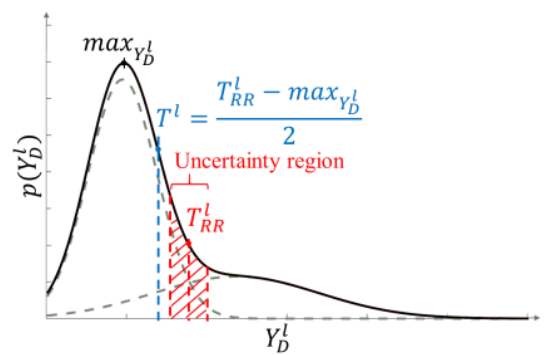

Fig. 7. Example of a $Y_{D}^{l}$ histogram with the selection of thresholds $T_{R R}^{l}$ and $T^{l}$.

For each pair of invariant features, a different $T^{l}$ is calculated. The selection criteria for the threshold accounts for the greater spectral variability of the $\omega_{n}$ class. Thus, a proper threshold selection requires the relaxation of the criteria of the state-of-the-art methods. In greater detail, $T^{l}$ should be selected in order to guarantee the presence of unchanged pixels covering the whole spectral range of samples from the study area yet avoiding changed samples. To this end, a combination of two stages is used: i) calculation of a reliable threshold value by means of a state-of-the-art method, and ii) definition of a more relaxed threshold. First, the optimal decision threshold $\left(\mathrm{T}_{R R}^{l}\right)$ according to the Bayesian decision theory is applied, considering a Rayleigh-Rice (RR) model [44], for each $l$ th most similar band in the feature space. Where RR model is a good approximation. Samples in $Y_{D}^{l}$ close to $\mathrm{T}_{R R}^{l}$ (see Fig. 7) are uncertain. Whereas pure unchanged pixels are needed to model the spectral relationship between $X_{1, a}$ and $X_{2, b}$ properly. Therefore, $\mathrm{T}_{R R}^{l}$ is refined to limit the presence of changed samples. The refinement process is conducted to satisfy the tradeoff between removing unchanged samples (small threshold values) and preserving the spectral variability (large threshold values) as:

$$
T^{l}=\frac{\mathrm{T}_{R R}^{l}-\max _{Y_{D}^{l}}}{2}, l=1, \ldots, 2 L
$$

where $\max _{Y_{D}^{l}}$ is the first maximum peak on the left of $\mathrm{T}_{R R}^{l}$ in the invariant feature space. Once $T^{l}$ is selected, $Y_{D}^{l}$ is thresholded to get candidate unchanged samples for the training set.

\section{Un-changed Samples Subsampling}

In order to avoid over-fitting and overload while performing the non-parametric regression, detected unchanged samples are sub-sampled by following two guidelines: i) select unchanged samples common to both spectral and textural features, and ii) select samples by preserving their statistical distribution. The previous guidelines are applied separately to each pair of invariant features. The sets of unchanged samples represent the variety of spectral relationships existing among the $L$ most similar bands from the spectral and textural viewpoint. The first guideline assures that samples are unchanged both from the spectral and textural point of view. Nevertheless, the number of training samples remains high. Thus, the second guideline aims at further reducing this number by preserving a uniform spatial distribution of the samples over the scene, as well as the statistical distribution of the original $X_{1, a}$ and $X_{2, b}$. For each pair of invariant features, the entire image is first divided into small blocks of size $R \times$ $R$, with $R<I<J$. Then a fraction of samples from each block is selected. On top of these samples, a maximum number of pixels $(Q)$, over all possible spectral values, are selected.

\section{Nonparametric Regression}

The last step of the proposed RRN approach is the nonparametric regression. The exact form of the nonlinear function $f$ in equation (1) does not need to be known explicitly prior to model training. In order to perform the nonparametric regression, different sets of training samples $T r_{l}$ $(l=1, \cdots, L)$ should be defined for each spectral channel to be predicted. Each of the $\operatorname{Tr}_{l}$ training sets is built by considering the positions given by the sub-sample step (previous section), but in this case, and as learnt from our preliminary work [45], invariant features are extracted from all the channels in $S_{1}$ and $S_{2}$ (remember that $B>A$ ). This is because several bands in $X_{2, b}$ may contribute to the estimation of the spectral information in each of the bands in $X_{1, a}$. Accordingly, the set of both spectral and spatial invariant features is defined as $X_{1}=\left\{X_{1, a, s p e}, X_{1, a, t x t}\right\}$ and $X_{2}=\left\{X_{2, b, s p e}, X_{2, b, t x t}\right\}$.

Given the above-described sets of $T_{l}$, and the sets of features $X_{1}$ and $X_{2}$, we model the temporal and spectral relationship between each $X_{1, a}$ and $X_{2} . X_{2}$ represents the input (source) and $X_{1, a}$ the output (target/reference). The method still works if $A>B$, by reversing the role of source and target images. The complexity of the relationship between $X_{1, a}$ and $X_{2}$ is captured by estimating $f(\cdot)$ with a non-parametric 
regression method. In this study we choose two nonparametric approaches namely ANN and SVR. However, any other non-parametric method can be used. Here we propose a novel configuration for the input and target training samples for predicting $\hat{X}_{1, a}$ (image acquired at $t_{2}$ by $S_{1}$ ) that accounts for the radiometric differences in the multisensor multitemporal dataset. $\hat{X}_{1, a}$ contains only the spectral channels, since our goal is only to homogenize the original images, though textural information is also available, but not predicted in the proposed architecture. A total of $A$ nonparametric regression models have to be trained, as limited by the sensor with lower number of bands, between $S_{1}$ and $S_{2}$. The target for each regression model is one of the spectral bands $X_{1, a, s p e}$ to be predicted. Whereas all the bands in $X_{2, b}$ are used as input to the $A$ regression models, i.e., $X_{2, b, s p e}$. As for the selection of training samples, the use of spectral information alone $\left(X_{2, b, s p e}\right)$ is not enough to model the relationship among $X_{1, a}$ and $X_{2, b}$. Thus, texture features are also used, where $X_{2, b, t x t}$ are extracted from $X_{2, b, s p e}$. Thus, a total of $2 \times B$ inputs are finally used for the derivation of the $A$ regression models. The inputs are always the same for all models, whereas the target varies on the bases of the channel to be estimated.

For ANN, a backpropagation architecture is considered. Equation (5) shows the non-parametric regression model for the training process. In (5), $H$ and $O$ stand for hidden and output layers, respectively; $\varphi_{H}(\cdot)$ and $\varphi_{O}(\cdot)$ represent the activation functions (e.g., linear, log-sigmoid, tan-sigmoid); and $w_{b j}$ and $w_{j k}$ represent the weights of the network connection in the hidden and output layers, respectively. $\beta_{H}$ and $\beta_{O}$ represent the bias introduced to the transfer functions. Once the model has been obtained, all the channels in $\hat{X}_{1, a}$ are predicted.

$$
X_{1, a}=\varphi_{O}\left(\beta_{O}+\sum_{j=1}^{J} w_{j k}\left(\varphi_{H}\left(\beta_{H}+\sum_{b=1}^{2 B} w_{b j} X_{2}\right)\right)_{j}\right) .
$$

For SVR, a $\epsilon$-SVR with a Radial Basis Function (RBF) kernel is used. Equation (6) shows the non-parametric regression model for the training process, where $\alpha_{i}$ and $\alpha_{i}^{*}$ represent the Lagrange multipliers of a quadratic estimation problem and $k(.,$.$) is a kernel function (e.g., homogeneous$ polynomial, RBF). Here RBF kernel is selected. Therefore, the hyper-parameter gamma $=\left(2 \sigma^{2}\right)^{-1}$ needs to be defined that corresponds to the width or scale of the kernel. Apart from the kernel parameters, $\epsilon$-SVR requires the definition of two parameters: i) $\epsilon$ (epsilon), the minimal required precision; and ii) $C$, the penalty associated with errors larger than $\epsilon$.

$$
X_{1, a}=\sum_{i=1}^{\left|T r_{l}\right|}\left(\alpha_{i}-\alpha_{i}^{*}\right) k\left(\left|T r_{l}\right|_{i}, X_{2}\right)
$$

The extension of the proposed method for the time series longer than two images is straightforward by selecting one image with the lowest number of spectral bands as reference/target.

\section{MUlTITEMPORAL INFORMATION EXTRACTION}

Several methods for MT information extraction can be found in the literature that may benefit of the proposed approach for the generation of homogeneous TS from MT multisensor VHR images. The selection of the method to derive MT information depends on the application itself. Here we consider $\mathrm{CD}$ as an option to demonstrate the effectiveness of the proposed approach. According to the-state-of-the-art, the CVA technique in a polar domain framework as proposed in [39] is used. To this end, the multispectral difference images $X_{D}$ is computed as:

$$
X_{D}=\hat{X}_{1, a}-X_{1, a}
$$

where $X_{D}$ is the magnitude of the change vectors. $\rho \in$ $\left[0, \rho_{\max }\right]$ (eq. (8)) carries information about presence/absence of changes. Small magnitude values are associated to no changes, whereas large values are associated to change. A threshold $T$ is commonly applied to separate changed from unchanged samples [39]. Here, the optimal $T$ is obtained according to the Bayesian decision theory by considering a Rayleigh-Rice (RR) model [44].

$$
\rho=\sqrt{\sum_{a=1}^{A}\left(X_{D, a}\right)^{2}}
$$

\section{EXPERIMENTAL RESULTS}

\section{A. Dataset Description and Multisensor Normalization}

In order to validate the proposed approach, pairs of VHR optical images acquired over an area located in the Trentino region in the north of Italy (Fig. 8) were selected. This area shows interesting properties from the orographic conformation and environmental variety point of view. Over a relatively small area it is possible to find: i) precious apple and vineyard fields; and ii) urban, sub-urban and industrial areas with different density and structure. Two multitemporal data sets were built over the sample area (yellow boxes in Fig. 8). Details are given in Table I. Dataset 2 is larger than dataset 1, and $t_{1}$ and $t_{2}$ images are acquired in the same year, but in different months. Whereas images in dataset 1 were acquired 4 years apart. Both datasets include an image with 4 spectral bands only. The dataset selection allows to test the robustness of the method when: i) poor spectral information is available, since this increases the complexity in modelling the relationship between $t_{1}$ and $t_{2}$; ii) the images are acquired in different seasons, which increases the spectral variability among images; iii) $A>B$, to show the capacity of the method to be extended to TS analysis; and iv) different kinds of land covers and land-cover changes exist. Therefore, the datasets show transitions among different phenological states in crop areas and transitions from vegetation to bare soil and/or to new man-made objects (i.e., construction of a new road and building).

Dataset 1 includes a QB image with four multispectral bands $(A=4)$, and a WV-2 one with eight $(B=8)$ bands. The spatial resolution of the QB image is $0.6 \mathrm{~m}$ for the panchromatic band and $2.4 \mathrm{~m}$ for multispectral bands, whereas WV-2 offers a higher spatial resolution in both panchromatic 


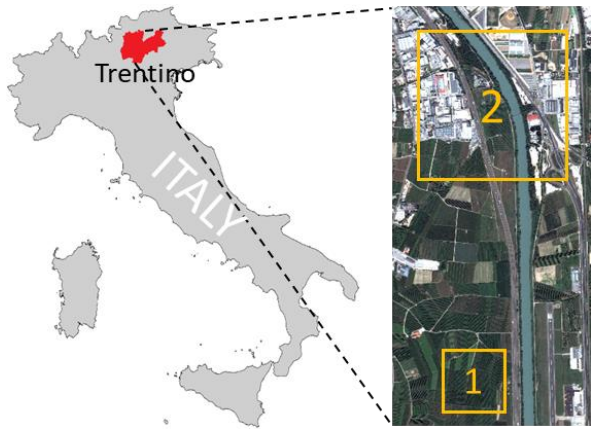

Fig. 8. Area of interest, Trentino region in the North of Italy.

and multispectral bands that is approximated to $0.5 \mathrm{~m}$ and $2 \mathrm{~m}$ by image providers, respectively. Dataset $2 t_{1}$ image is a WV2 image with 8 multispectral bands $(A=8)$, whereas $t_{2}$ is a GE-1 image with four bands $(B=4)$. The spatial resolution of the WV-2 and GE-1 images is $0.5 \mathrm{~m}$ for the panchromatic band and $2.0 \mathrm{~m}$ for multispectral bands (as approximated by image providers). Table II summarizes the characteristics of $\mathrm{QB}$, WV-2 and GE-1 images from the spectral and spatial point of view. The spatial resolution differences in dataset 1 imply that the sizes $I_{1} \times J_{1}$ and $I_{2} \times J_{2}$ of the two images are different despite they cover the same surface. Thus, pixel-by-pixel comparison cannot be directly applied since the same pixel coordinates in the two images do not correspond to the same position on the ground. Concerning spectral domain, we canobserve that the four primary multi-spectral bands of $\mathrm{QB}$, WV-2 and GE-1 are acquired over similar spectral ranges (e.g., red), but not identical (e.g., NIR). Similar considerations hold for blue and green bands. Given that bands for the three sensors fall into the same ranges, they can be compared and used to perform the regression. For both dataset the reference image is the one with the smaller number of bands (i.e., $t_{1}$ for dataset 1 and $t_{2}$ for dataset 2). This is because from the physical viewpoint, it is not possible to predict $A$ bands from just $B$ if $A>B$. In order to apply the proposed method for generation of homogeneous VHR TS, multisensory

TABLE I. DATASETS DESCRIPTION.

\begin{tabular}{|c|c|c|c|c|}
\hline & \multicolumn{2}{|c|}{ Dataset 1} & \multicolumn{2}{|c|}{ Dataset 2} \\
\hline & $t_{1}$ & $t_{2}$ & $t_{1}$ & $t_{2}$ \\
\hline Sensor & QB & WV-2 & WV-2 & GE-1 \\
\hline $\begin{array}{c}\text { Acquisition } \\
\text { date }\end{array}$ & $\begin{array}{l}\text { July } \\
2006\end{array}$ & $\begin{array}{c}\text { August } \\
2010\end{array}$ & $\begin{array}{l}\text { May } \\
2011\end{array}$ & $\begin{array}{c}\text { September } \\
2011\end{array}$ \\
\hline $\begin{array}{c}\text { Off-nadir } \\
\text { angle }\end{array}$ & $14.1^{\circ}$ & $19.3^{\circ}$ & $7.8^{\circ}$ & $14.4^{\circ}$ \\
\hline Dimensions & \multicolumn{2}{|c|}{1024 x 1024 pixels } & \multicolumn{2}{|c|}{$1800 \times 1800$ pixels } \\
\hline
\end{tabular}

TABLE II. MAIN CHARACTERISTICS OF QUICKBIRD, WORLDVIEW-2 AND GEOEYE-1 OPTICAL SENSORS [14].

\begin{tabular}{cccc}
\hline Satellite & QuickBird & WorldView-2 & GeoEye-1 \\
\hline & $445-900$ (pan) & $450-800$ (pan) & $450-800$ (pan) \\
& & $400-450$ (coastal) & \\
& $450-520$ (blue) & $450-510$ (blue) & $450-510$ (blue) \\
& $520-600$ (green) & $510-580$ (green) & $510-580$ (green) \\
Bands & & $585-626$ (yellow) & \\
(nm) & $630-690$ (red) & $630-690$ (red) & $655-690$ (red) \\
& & $705-745$ (red edge) & \\
& & $770-895($ NIR 1$)$ & $780-920(\mathrm{NIR})$ \\
& $760-900(\mathrm{NIR})$ & $860-1040(\mathrm{NIR} 2)$ & $0.41 \mathrm{~m}$ \\
Spatial & $0.61 \mathrm{~m}$ & $0.46 \mathrm{~m}$ & $1.65 \mathrm{~m}$ \\
\hline
\end{tabular}

homogenization and RRN were applied.

Multisensor homogenization was performed by: i) absolute radiometric normalization and ii) geometric normalization. For ARN step, all images were provided by DigitalGlobe Foundation in the context of the "MS-TS - Analysis of Multisensor VHR image Time Series" project [46]. Here, the ARN consists on the conversion from DNs to TOA, which was conducted before delivery by means of the Atmospheric Compensation (AComp) algorithm, which corrects for the scattering and absorption effects in the atmosphere [3], [47], [48]. Given the orography of the study area and the possible distortions, we applied orthorectification by using a DEM (1m) obtained from LiDAR data [49]. Additional pixel-topixel problems are also observed due to differences in the view angle, and co-registration should be applied. In order to achieve a better co-registration, PanSharpening (PS) was applied by means of the Gram-Schmidt method [50]. Here ENVI software package was employed [51].

Co-registration of the two-dataset pairs, covering the study area in Fig. 8, was conducted by using a polynomial function of second order. For Dataset 1, 79 uniformly distributed Ground Control Points (GCP) were selected. Whereas 68 uniformly distributed GCP were selected for Dataset 2. For Dataset 1, the WV-2 image was resampled during coregistration in order to be able to pansharpen multisensor VHR QB and WV-2 images after preliminary normalization. Dataset 1 has a spatial resolution of $0.6 \mathrm{~m}$ and a size of $1024 \times 1024$, whereas Dataset 2 has a spatial resolution of $0.5 \mathrm{~m}$ and a size of $1800 \times 1800$. In order to perform quantitative analysis, a reference map for dataset 1 was defined by photointerpretation and a priori knowledge on the scene (Fig. 9 third column), showing 706364 unchanged pixels (black color) and 334469 changed pixels (white color). For dataset 2, considering the extent of the area and the fact that we have no complete knowledge of the changes occurred on the ground, it was not possible to derive an exhaustive reference map. Thus, quantitative analysis was based on 121357 samples marked as changed, and 120445 as unchanged, selected by photointerpretation. For comparison purposes, a multitemporal false color composition of the dataset is provided in Fig. 9.f, where green and magenta colors represent changes.

TABLE III. PAIR OF MOST SIMILAR BANDS FOR QB, WV-2 AND GE-1; AND HISTOGRAM COMPENSATION VALUE ( $P$ ) FOR (A) DATASET 1 AND (B) 2.

\begin{tabular}{ccc}
\hline & $(\mathrm{A})$ \\
\hline QB $(\mathrm{nm})$ & WV-2 (nm) & $\mathrm{P}$ \\
\hline $450-520(\mathrm{~B} 1)$ & $450-510(\mathrm{~B} 2)$ & 0.020 \\
$520-600(\mathrm{~B} 2)$ & $510-580(\mathrm{~B} 3)$ & 0.016 \\
$630-690(\mathrm{~B} 3)$ & $630-690(\mathrm{~B} 5)$ & 0.023 \\
$760-900(\mathrm{~B} 4)$ & $770-895(\mathrm{~B} 7)$ & 0.145 \\
\hline \multicolumn{3}{c}{$(\mathrm{B})$} \\
\hline WV-2 (nm) & GE-1 (nm) & $\mathrm{P}$ \\
450-510 (B2) & $450-510(\mathrm{~B} 1)$ & 0.024 \\
$510-580(\mathrm{~B} 3)$ & $510-580(\mathrm{~B} 2)$ & 0.020 \\
$630-690(\mathrm{~B} 5)$ & $655-690(\mathrm{~B} 3)$ & 0.015 \\
$770-895(\mathrm{~B} 7)$ & $780-920(\mathrm{~B} 4)$ & 0.043 \\
\hline
\end{tabular}

\section{B. Relative Radiometric Normalization and Design of Experiments}

Once the preliminary multisensor normalization is achieved, we move to the proposed RRN. The first step is to select invariant features among the two sensors. To this end, a total 

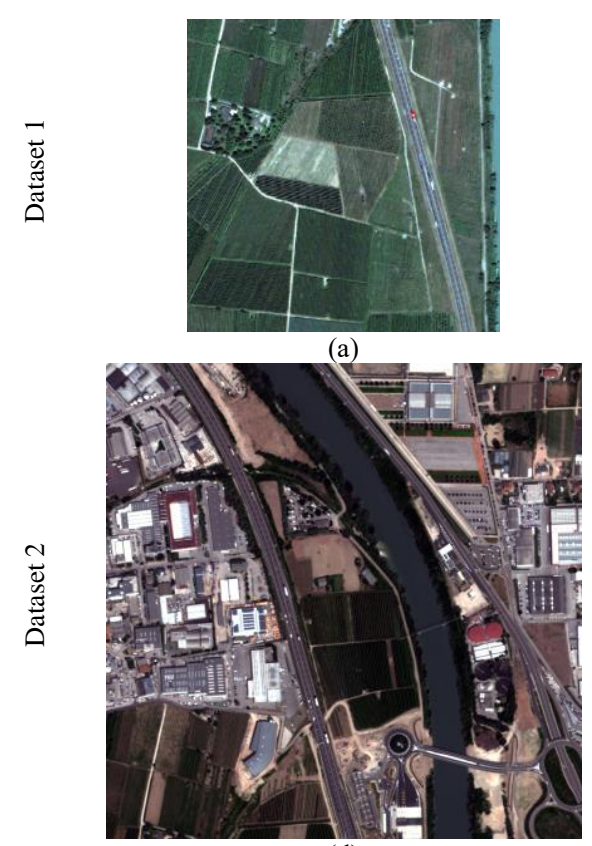

(d)

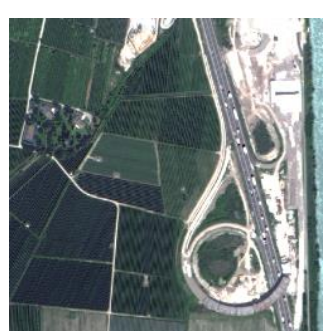

(b)

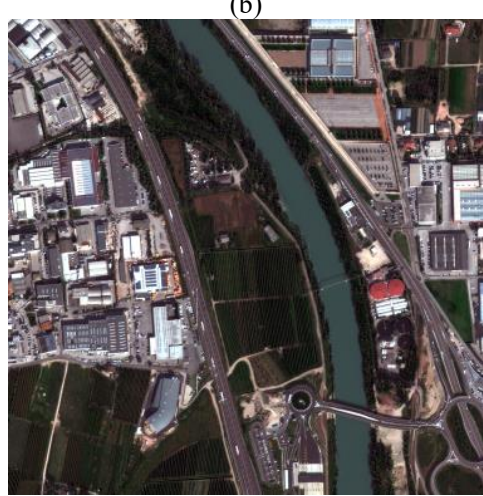

(e)

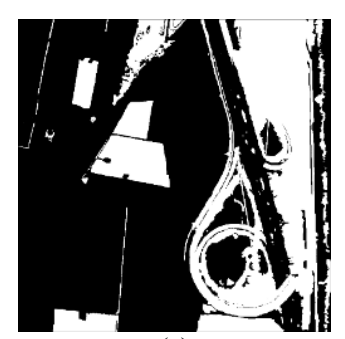

(c)

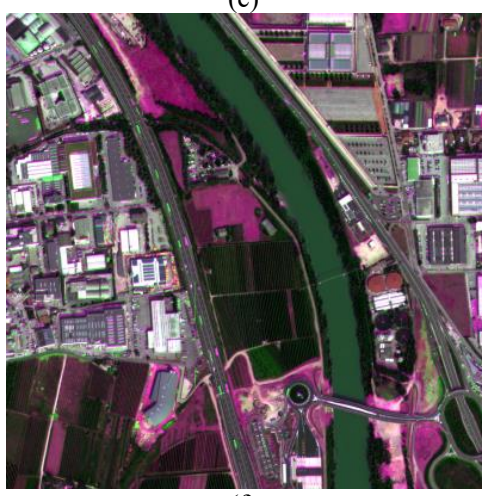

(f)

Fig. 9. True color composition of the pansharpened (a) QB image acquired in July 2006 and (b) WV-2 image acquired in August 2010, and (c) reference map (data set 1). True color composition of the pansharpened (d) WV-2 image acquired in May 2011, (e) GE-1 image acquired in September 2011, and (f) multitemporal false color composition (red: red band WV2 2011 image, green: green GE1 2011 image, and blue: blue WV2 2011 image) (data set 2).

TABLE IV. THRESHOLD $\left(T^{l}\right)$ AND NUMBER OF TRAINING SAMPLES BEFORE $\left(\left|Y_{D}^{l}<T^{l}\right|\right)$ AND AFTER $\left(\left|T r_{l}\right|\right)$ SUB-SAMPLING FOR DATASETS (A) 1 AND (B) 2.

\begin{tabular}{cc|ccc}
\hline Band & Feature & $\boldsymbol{T}^{\boldsymbol{l}}$ & $\left|\boldsymbol{Y}_{\boldsymbol{D}}^{\boldsymbol{l}}<\boldsymbol{T}^{\boldsymbol{l}}\right|$ & $\left|\boldsymbol{T r}_{\boldsymbol{l}}\right|$ \\
\hline \multirow{2}{*}{ Blue } & Spectral & 0.012 & 616739 & \multirow{2}{*}{4998} \\
& Texture & 0.040 & 242034 & \\
\hline \multirow{2}{*}{ Green } & Spectral & 0.013 & 560094 & \multirow{2}{*}{5436} \\
& Texture & 0.030 & 158496 & \\
\hline \multirow{2}{*}{ Red } & Spectral & 0.014 & 528475 & \multirow{2}{*}{6155} \\
& Texture & 0.030 & 161157 & \\
\multirow{2}{*}{ NIR } & Spectral & 0.025 & 265267 & \multirow{2}{*}{8980} \\
& Texture & 0.030 & 157491 & \\
\hline \multirow{5}{*}{ Band } & Feature & $(\mathrm{B})$ & & \\
\hline \multirow{2}{*}{ Blue } & Spectral & 0.023 & 1927967 & \multirow{2}{*}{12401} \\
& Texture & 0.030 & 640193 & \\
\hline \multirow{2}{*}{ Green } & Spectral & 0.023 & 1822823 & \multirow{2}{*}{11897} \\
& Texture & 0.030 & 632969 & \\
\hline \multirow{2}{*}{ Red } & Spectral & 0.030 & 1474649 & \multirow{2}{*}{12221} \\
\hline \multirow{2}{*}{ NIR } & Texture & 0.031 & 634457 & \\
& Spectral & 0.034 & 1457945 & \multirow{2}{*}{13458} \\
\hline
\end{tabular}

of $L=4$ most similar bands among $S_{1}$ and $S_{2}$ were first identified (see Table III). Then, spectral and textural features were extracted. For spectral features, the $L=4$ pairs of most similar bands were compensated based on histogram shift value $P$ (see Table III). WV-2 image is homogenized to QB and GE-1, respectively. In the case of textural features, wellknown state-of-the-art features, such as Gray Level Cooccurrence Matrix (GLCM) [52] and Gabor ones [53], were tested under different configurations. GLCM-contrast showed the best results in terms of normalization and CD results and was thus selected as the invariant feature.

The next steps are the selection of unchanged samples and their sub-sampling. The first step applies the absolute UID asin (3), and then $T^{l}$ is calculated as in (4). The $T^{l}$ values for each invariant features (spectral and textural), as well as the number of training samples before $\left(\left|Y_{D}^{l}<T^{l}\right|\right)$ and after $\left(\left|\operatorname{Tr}_{l}\right|\right)$ the sub-sampling step are given in Table IV for both datasets. After some trials the size of the blocks for the spatial reduction was set to $R=64$ and $R=128$, for datasets 1 and 2 , respectively. The fraction of training samples for dataset 1 was of $30 \%$, whereas for dataset 2 the fraction was of $10 \%$. When no samples are found in the block, no operation is performed. A maximum of $Q=100$ samples per reflectance value (with a precision of 3) over the whole spectral range were selected for each dataset. The selection of these parameters is related to: i) the computational power and ii) the conservation of spectral variability to model the relationship between $S_{1}$ and $S_{2}$. Large numbers result in the selection of more samples, which in turn results in a higher computational requirement and a higher probability of overfitting while applying the non-parametric regression. Lower numbers result in the selection of less samples, which in turn results in lower computational requirements and poorer spectral representation to model the $S_{1}$ and $S_{2}$ relationship.

The last step of the proposed RRN is the non-parametric regression. Here we choose two methods (ANN and SVR) to demonstrate the robustness of the proposed approach against the non-linear regression algorithm. However, any other nonparametric method can be used. A total of 4 regression models were derived, one for each couple of most similar bands. The input data corresponded to the spectral and textural features of the WV-2 images, for a total of 16 input variables, whereas the target corresponded to the specific spectral band to be predicted in QB or GE-1. Final configuration for ANN and SVR were the same for both datasets.

1. For the $A N N$, i) one input layer with 16 neurons (one for each WV-2 spectral and textural bands), ii) three hidden layers with 15,8 and 10 neurons having tan-sigmoid, logsigmoid and pure-line activation functions, respectively, and iii) one output layer with one neuron having a linear 
TABLE V. FinAL MSE AND COMPUTATIONAL TIME OF ANN AND SVR FOR DATASETS 1 AND 2.

\begin{tabular}{|c|c|c|c|c|c|c|c|c|}
\hline \multirow{3}{*}{$\begin{array}{c}\text { Spectral } \\
\text { Band }\end{array}$} & \multicolumn{4}{|c|}{ Dataset 1} & \multicolumn{4}{|c|}{ Dataset 2} \\
\hline & \multicolumn{2}{|c|}{ ANN } & \multicolumn{2}{|c|}{ SVR } & \multicolumn{2}{|c|}{ ANN } & \multicolumn{2}{|c|}{ SVR } \\
\hline & $\operatorname{MSE}\left(10^{-5}\right)$ & Time & $\operatorname{MSE}\left(10^{-5}\right)$ & Time & $\operatorname{MSE}\left(10^{-5}\right)$ & Time & $\operatorname{MSE}\left(10^{-5}\right)$ & Time \\
\hline Blue & 2.2190 & & 3.6822 & $0.073 \mathrm{~h}$ & 9.0272 & & 9.1273 & $25.284 \mathrm{~h}$ \\
\hline Green & 3.2567 & $001,02 \mathrm{~b}$ & 4.5511 & $0.452 \mathrm{~h}$ & 10.709 & $0102 \mathrm{~h}$ & 11.410 & $21.149 \mathrm{~h}$ \\
\hline Red & 4.9059 & $0.01-0.2 \mathrm{~h}$ & 6.0398 & $1.446 \mathrm{~h}$ & 19.176 & $0.1-0.2 \mathrm{~h}$ & 18.034 & $24.250 \mathrm{~h}$ \\
\hline NIR & 15.192 & & 16.721 & $9.006 \mathrm{~h}$ & 28.709 & & 30.689 & $40.435 \mathrm{~h}$ \\
\hline
\end{tabular}

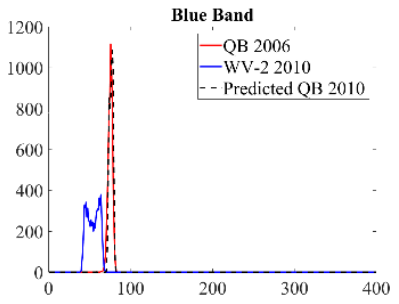

(a)

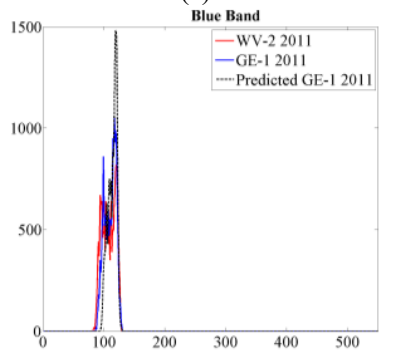

(e)

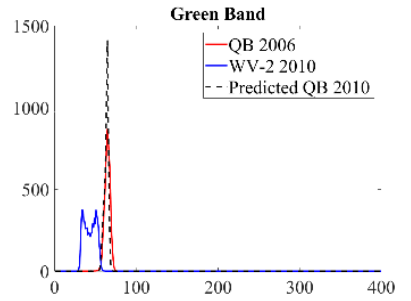

(b)

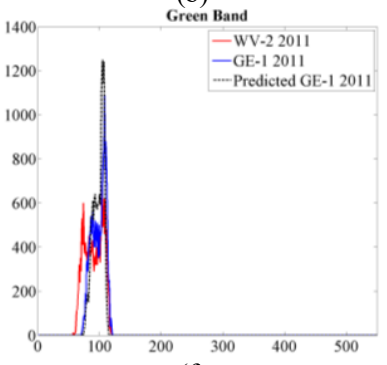

(f)

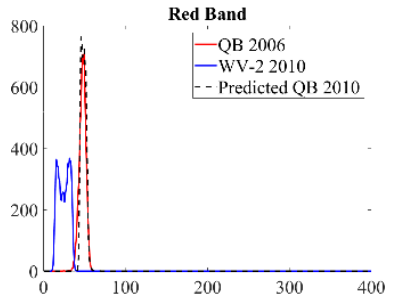

(c)

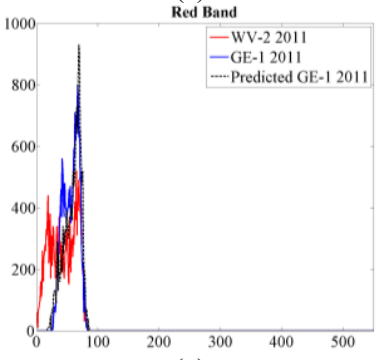

(g)

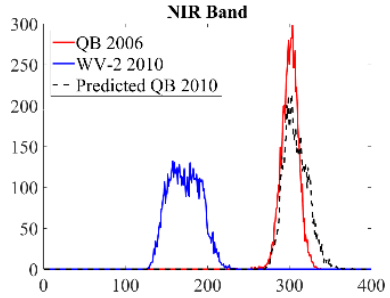

(d)

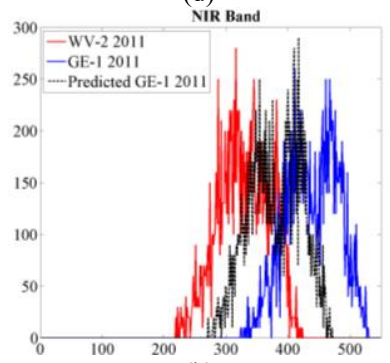

(h)

Fig. 10. Histograms for blue, green, red, and NIR bands of unchanged areas for $\mathrm{X}_{1,1}, \mathrm{X}_{2, \mathrm{l}}$ and $\widehat{\mathrm{X}}_{1, \mathrm{a}_{\mathrm{ANN}}}$ : (Top) data set 1 and (Bottom) data set 2 .

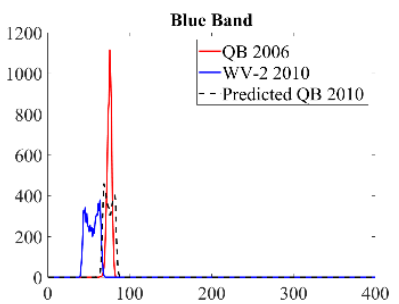

(a)

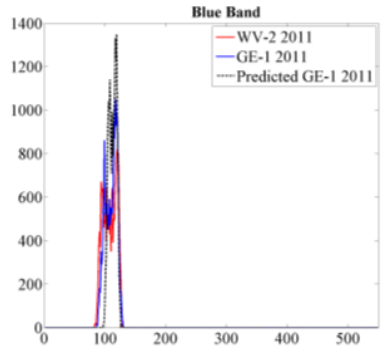

(e)

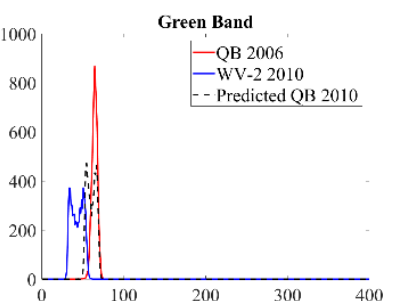

(b)

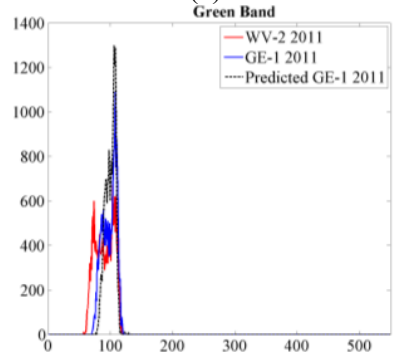

(f)

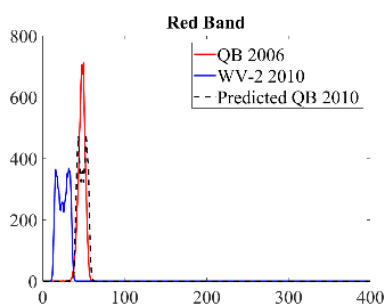

(c)

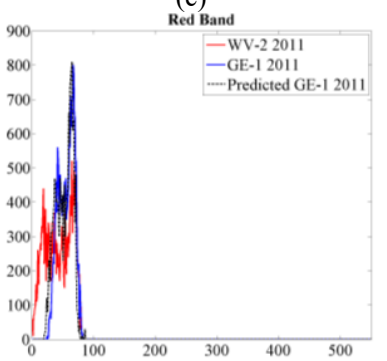

(g)

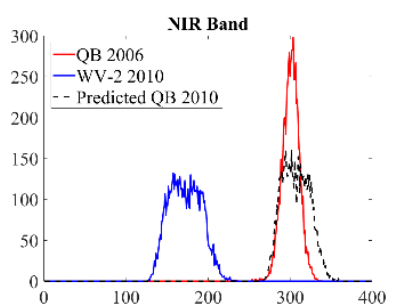

(d)

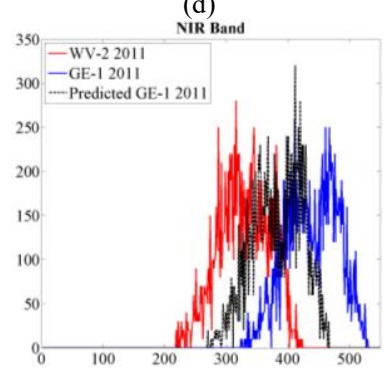

(h)

Fig. 11. Histograms for blue, green, red, and NIR bands of unchanged areas for $X_{1, l}, X_{2, l}$ and $\widehat{X}_{1, a_{S V R}}$ : (Top) data set 1 and (Bottom) data set 2.

activation function, were used. The number of neurons in the input and output layers are the same as the number of input and output features. The best configuration for the number of hidden layers and their neurons was selected by cross-validation based on the Mean Squared Error (MSE). Ranges for the parameters were: number of hidden layers = $[1,3]$ and number of neurons $=[8,20]$.

2. For the SVR, a similar configuration as for ANN was held, with 16 variables for the input and one variable for the target. An RBF kernel was used and the corresponding parameters for the training process were obtained by cross- validation process. Ranges for the parameters were: $C=$ $[10,500]$, gamma $=[0.1,3.0]$ and $\epsilon=[0.01,0.1]$.

The MSE calculated between the QB image $X_{1, a}\left(t_{1}\right)$ and the predicted QB $\hat{X}_{1, a}\left(t_{2}\right)$ (Dataset 1) and the GE-1 image $X_{2, b}\left(t_{2}\right)$ and the predicted GE-1 $\hat{X}_{2, b}\left(t_{1}\right)$ (Dataset 2 ) by the 4 ANNs and the 4 SVRs are provided in Table V together with computational time. In both cases, the training time includes both training and cross-validation. The computational time is the one obtained by using MATLAB $\AA$ on a standard workstation with Intel(R) Xeon(R) CPU @3.40 GHz, 16.00 
GB RAM. The MSE is quite similar for the two nonparametric regression methods, but the time required for the training process differs significantly. In the case of SVR, it depends on the number of training samples. Comparing $\left|T r_{l}\right|$ shown in Table IV with the training times in Table V, we can easily conclude that the training time increases with $\left|\operatorname{Tr}_{l}\right|$ in a nonlinear way.

The performance of the proposed approach was evaluated by qualitative and quantitative analysis. In the qualitative case, histograms were extracted over unchanged areas to assess the similarity of original and predicted images. In the quantitative case, two approaches are used: i) calculation of KullbackLeibler (KL) distance between the histograms of unchanged areas [54] and; ii) MT information extraction, change detection by means of CVA-magnitude thresholding. In the $\mathrm{KL}$ distance, we expect histograms from the predicted images to be closer to those of the reference ones. For the CD based validation, three experiments were designed: i) experiment 1 (exp. 1) applies CVA to the ARN images $\left(X_{1, a}\right.$ and $\left.X_{2, b}\right)$, ii) experiment 2 (exp. 2) applies CVA to the original image $\left(X_{1, a}\right)$ and predicted image obtained with $\operatorname{ANN}\left(\hat{X}_{1, a_{A N N}}\right)$; and iii) experiment 3 (exp. 3) applies CVA to the original image $\left(X_{1, a}\right)$ and predicted image obtained with $\operatorname{SVR}\left(\widehat{X}_{1, a_{S V R}}\right)$. In exp. 2 and 3 , the four spectral bands are used for computing the magnitude variable (see eq. (8)). Whereas in the case of exp. 1 the most similar bands are used. Other experiments with standard RRN methods were also performed, but the results are not reported in the paper given their poor performance.

\section{MT information extraction and experimental results}

For the qualitative assessment, histograms of unchanged areas were compared. It is expected that after prediction, $\hat{X}_{1, a}$ histograms become closer to $X_{1, a}$ than to $X_{2, b}$, both in shape and position for dataset 1 , and $\hat{X}_{2, b}$ histograms become closer to $X_{2, b}$ than to $X_{1, a}$ for dataset 2 . We also expect to have higher similarity in the case of dataset 1 , since it shows less seasonal differences. The histograms in Fig. 10 and Fig. 11 confirm these expectations. For each most similar spectral pair, the histograms of the spectral bands predicted from the WV-2 image by ANN and SVR (dashed black lines in Fig. 10 and Fig. 11) have behaviors more similar to the original reference images rather than to the $\mathrm{WV}-2$ ones. In the specific case of ANN histograms, they are more similar to the original reference images, both in shape and position, though less obvious for the NIR band in dataset 2. In the case of SVR histograms, one can see that the predicted images have changed their histogram in shape and position, but not as much as in the ANN case. The problem with NIR band in dataset 2 remains as for ANN case. The main difference between ANN and SVR results relies in the fact that SVR is not able to completely model the shape of the histogram, which remains still more similar to that of the original WV-2 image. Even though the proposed approach mitigates most of the intrinsic differences arising from the complexity of working with MT multisensor VHR images, some differences in shape and position still remain.
The above is quantitatively corroborated by the KL distance shown in Table VI. As expected, the predicted image is always closer to the target/reference image one rather than to the original one. Furthermore, ANN generates, in general, smaller distances than SVR. Since it results in a better prediction, from the histogram perspective. In the specific case of dataset 2, Table VI further corroborates the robustness of the method to: i) the use of images acquired in different seasons, ii) the availability of less spectral information (due to acquisitions in the same year); and iii) the capability of the method to operate under the condition where $A>B$.

For the quantitative analysis, $C D$ maps are derived by means of CVA and as defined in exp. 1, 2 and 3, by means of equation (8). Areas corresponding to radiometric changes were extracted by thresholding the magnitude variable. $T$ was automatically selected by applying the method in [44]. The $T$ values for exp. 1, 2 and 3 for dataset 1 were $0.135,0.094$ and 0.126 ; and $0.090,0.067$ and 0.074 for dataset 2 . A comparison of the CD maps with the reference maps (see Fig. 12 and Fig. 13) pointed out the improvement achieved when working with the proposed approach, both in ANN and SVR regression cases, specifically on the reduction of False Alarms (FA) and Missed Alarms (MA) for both datasets. In the case of dataset 1 , improvements are related to the identification of the two specific changes located in the central part of the study area, which were not identified before applying the proposed homogenization. Whereas in the case of dataset 2 , the improvements are related to the reduction of FA coming from the river (which did not change) and to a better detection of the changes from-to bare soil-vegetation and from buildings.
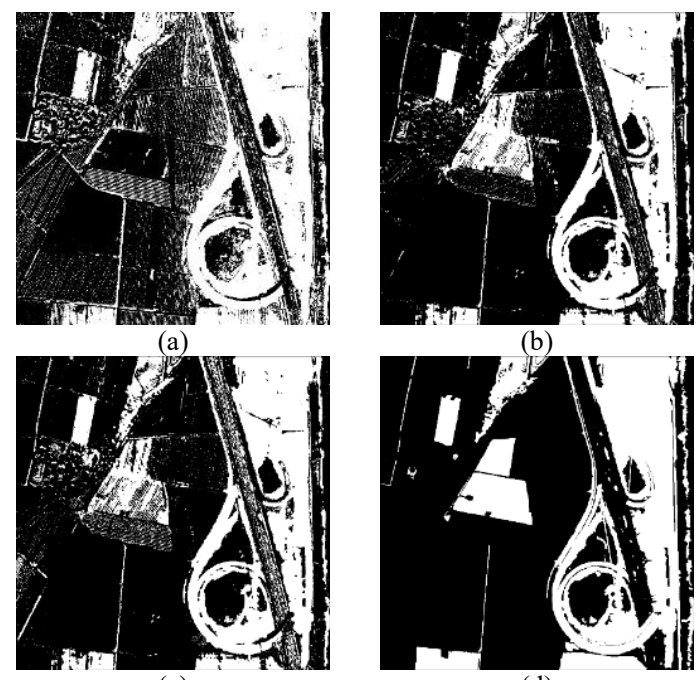

(c)

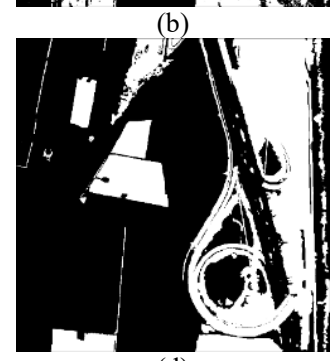

(d)

Fig. 12. Binary CD maps obtained by CVA for a) exp. 1, b) exp. 2 and c) exp. 3. d) Reference map (dataset 1).

Table VII shows the quantitative results, where for dataset 1 the number of FA identified by exp. 1 decreased almost of $67 \%$ with ANN homogenization and $61 \%$ with SVR one. These results are reflected in the OA, where exp. 2 gained about $11 \%$ over exp. 1 and $1 \%$ over exp. 3; and exp. 3 gained about $10 \%$ over exp. 1. For dataset 2 the number of FA decreased of about $65 \%$ in exp. 2 and $42 \%$ in exp. 3. This results in the increase of the $\mathrm{OA}$ for both experiments 


\begin{tabular}{c|cc|cc|cc|cc}
\multicolumn{3}{c|}{ TABLE VI. KL DISTANCE BETWEEN THE HISTOGRAMS OF UNCHANGED AREAS FOR DATASETS 1 AND 2.} \\
\hline \multirow{2}{*}{ Spectral } & \multicolumn{3}{|c|}{ Dataset 1} & \multicolumn{3}{c|}{ Dataset 2} \\
\cline { 2 - 9 } Band & \multicolumn{2}{|c|}{$\hat{X}_{1, a_{A N N}}$} & \multicolumn{2}{|c|}{$\hat{X}_{1, a_{S V R}}$} & \multicolumn{2}{|c|}{$\hat{X}_{1, a_{A N N}}$} & \multicolumn{2}{|c}{$\hat{X}_{1, a_{S V R}}$} \\
\cline { 2 - 9 } & $X_{1, l}$ & $X_{2, l}$ & $X_{1, l}$ & $X_{2, l}$ & $X_{1, l}$ & $X_{2, l}$ & $X_{1, l}$ & $X_{2, l}$ \\
\hline Blue & 2.65 & 83.43 & 11.87 & 69.05 & 1.90 & 0.86 & 2.77 & 1.62 \\
Green & 3.73 & 79.76 & 9.55 & 55.20 & 2.34 & 0.87 & 2.88 & 0.96 \\
Red & 3.36 & 81.66 & 3.44 & 77.94 & 2.03 & 0.35 & 2.02 & 0.51 \\
NIR & 3.78 & 63.26 & 5.16 & 62.50 & 2.87 & 3.68 & 3.30 & 3.52 \\
\hline
\end{tabular}

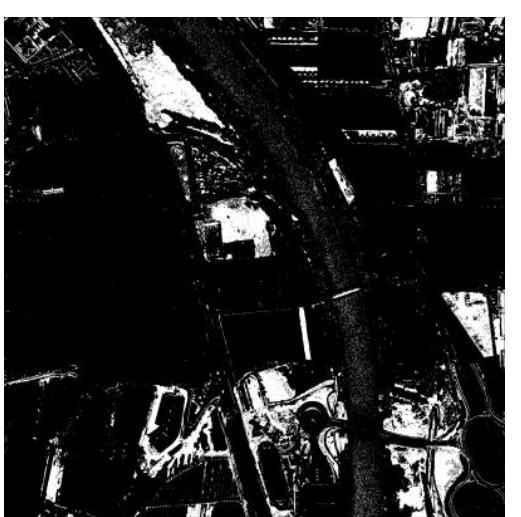

(a)

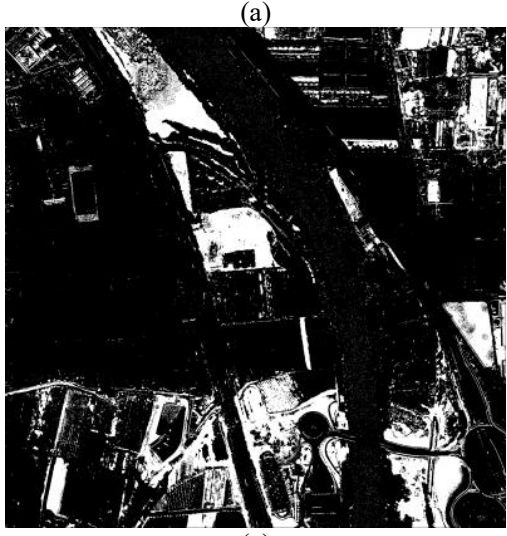

(c)

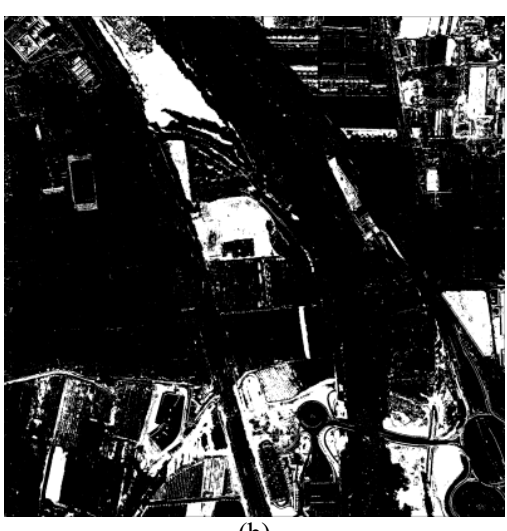

(b)

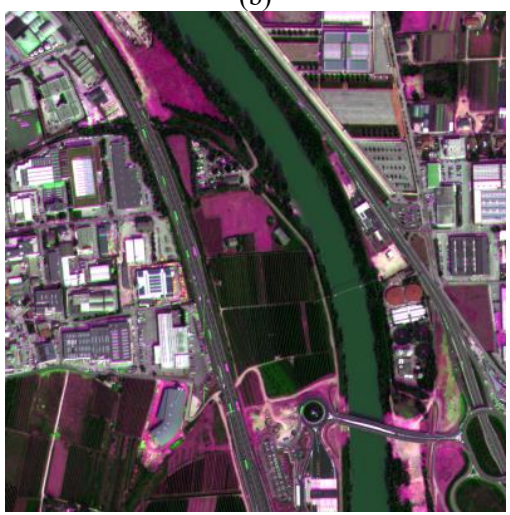

(d)

Fig. 13. Binary CD maps obtained by CVA for a) exp. 1, b) exp. 2 and c) exp. 3. d) Reference map (dataset 2).

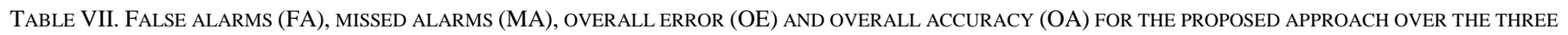
EXPERIMENTS (DATASETS 1 AND 2).

\begin{tabular}{c|cccc|cccc}
\hline \multirow{2}{*}{ Exp. } & \multicolumn{4}{|c|}{ Dataset 1 } & \multicolumn{3}{c}{ Dataset 2 } \\
\cline { 2 - 9 } & FA & MA & OE & OA (\%) & FA & MA & OE & OA (\%) \\
\hline 1 & 145477 & 68659 & 214136 & 79.58 & 3344 & 32662 & 36006 & 85.11 \\
2 & 47210 & 52046 & 99256 & 90.53 & 965 & 11388 & 12353 & 94.89 \\
3 & 56383 & 57454 & 113837 & 89.14 & 1638 & 18903 & 20541 & 91.50 \\
\hline
\end{tabular}

involving the proposed homogenization procedure for multisensor multitemporal images. Exp. 2 gained about $9 \%$ over exp. 1 and $3 \%$ over exp. 3; and exp. 3 gained about $6 \%$ over exp. 1. As expected, the OA increase from exp. 1 to exp. 2 and 3 is lower than in dataset 1 due to differences in the seasonal acquisition. Yet the proposed approach is able to improve the $\mathrm{CD}$ results. In overall, the results prove the effectiveness of the proposed homogenization approach of multisensor MT VHR images. Furthermore, based on the qualitative and quantitative analysis, as well as in terms of computational time, for the considered images ANN is slightly better than SVR. However, other regression methods can be considered.

\section{CONCLUSION}

In this paper, a novel method for generating homogenous VHR TS based on non-parametric regression of multisensor bitemporal optical images has been proposed. The proposed method effectively normalizes the multisensor multitemporal images by combining a preliminary normalization (based on ARN) and a RRN method. The proposed RRN method is based on a novel CD-driven approach for RCSS selection and a non-parametric regression model. In RRN, a prediction is achieved by deriving single band models with non-parametric regressions (ANN and SVR) that allows to capture the spatial/spectral variability of VHR multisensor multitemporal images which is usually not guaranteed in the state-of-the-art approaches for both radiometric normalization and/or change 
detection. The models allowed us to predict all the spectral bands for the $X_{1, a}$ image, as they would be acquired at $t_{2}$ by $S_{1}$, based on the original $X_{2, b}$ image bands and the reference un-changed pixels. The proposed CD-driven approach for training samples selection showed to be effective from the MSE view point, as well as from the MT information extraction one. Experimental results on real datasets, made-up of VHR bi-temporal and multisensor optical images, confirmed the effectiveness of the proposed approach and the improvement in multitemporal analysis that can be achieved by using the predicted images over the preliminary normalized ones. A major improvement is observed when ANN predicted image is used, but a good improvement is visible while using SVR predicted one as well. The selection of other spectral and/or textural features for the training stage could be considered to further improve the current CD OA. A further analysis can be carried on by using Deep Neural Network nonparametric regression to achieve both the normalization and $\mathrm{CD}$ processes at the same time.

\section{ACKNOWLEDGMENT}

This research is being developed under the project "MS-TS - Analysis of Multisensor VHR image Time Series" with DigitalGlobe Foundation. Authors would like to thank DigitalGlobe Foundation for data crosscheck and images provided for the research development.

\section{REFERENCES}

[1] Y. T. Solano-Correa, F. Bovolo, and L. Bruzzone, "Change detection in very high resolution multisensor optical images," 2014, vol. 9244, pp. 924410-924410-13.

[2] Y. T. Solano-Correa, F. Bovolo, and L. Bruzzone, "An Approach for Unsupervised Change Detection in Multitemporal VHR Images Acquired by Different Multispectral Sensors," Remote Sens., vol. 10, no. 4, p. 533, Mar. 2018.

[3] L. Bruzzone and F. Bovolo, "A Novel Framework for the Design of Change-Detection Systems for Very-High-Resolution Remote Sensing Images," Proc. IEEE, vol. 101, no. 3, pp. 609-630, Mar. 2013.

[4] F. Pacifici, N. Longbotham, and W. J. Emery, "The Importance of Physical Quantities for the Analysis of Multitemporal and Multiangular Optical Very High Spatial Resolution Images," IEEE Trans. Geosci. Remote Sens., vol. 52, no. 10, pp. 62416256, Oct. 2014.

[5] F. Bovolo, L. Bruzzone, and Y. T. Solano-Correa, "Multitemporal Analysis of Remotely Sensed Image Data," in Comprehensive Remote Sensing, S. Liang, Ed. Oxford: Elsevier, 2018, pp. 156-185.

[6] M. N. Klaric et al., "GeoCDX: An Automated Change Detection and Exploitation System for High-Resolution Satellite Imagery," IEEE Trans. Geosci. Remote Sens., vol. 51, no. 4, pp. 2067-2086, Apr. 2013.

[7] G. Hong and Y. Zhang, "A comparative study on radiometric normalization using high resolution satellite images," Int. J. Remote Sens., vol. 29, no. 2, pp. 425-438, Jan. 2008.

[8] B. Zitová and J. Flusser, "Image registration methods: a survey," Image Vis. Comput., vol. 21, no. 11, pp. 977-1000, Oct. 2003.

[9] J. Inglada and A. Giros, "On the possibility of automatic multisensor image registration," IEEE Trans. Geosci. Remote Sens., vol. 42, no. 10, pp. 2104-2120, Oct. 2004.

[10] Y. Han, F. Bovolo, and L. Bruzzone, "An Approach to Fine Coregistration Between Very High Resolution Multispectral
Images Based on Registration Noise Distribution," IEEE Trans. Geosci. Remote Sens., vol. 53, no. 12, pp. 6650-6662, Dec. 2015.

[11] Y. Han, F. Bovolo, and L. Bruzzone, "Edge-Based Registration-Noise Estimation in VHR Multitemporal and Multisensor Images," IEEE Geosci. Remote Sens. Lett., vol. 13, no. 9, pp. 1231-1235, Sep. 2016.

[12] A. Wong and D. A. Clausi, "ARRSI: Automatic Registration of Remote-Sensing Images," IEEE Trans. Geosci. Remote Sens., vol. 45, no. 5, pp. 1483-1493, May 2007.

[13] Y. Han, J. Choi, Y. Byun, and Y. Kim, "Parameter Optimization for the Extraction of Matching Points Between High-Resolution Multisensor Images in Urban Areas," IEEE Trans. Geosci. Remote Sens., vol. 52, no. 9, pp. 5612-5621, Sep. 2014.

[14] C. Persello, "Advanced Techniques for the Classification of Very High Resolution and Hyperspectral Remote Sensing Images," PhD, University of Trento, Trento, 2010.

[15] A. A. Nielsen, "The Regularized Iteratively Reweighted MAD Method for Change Detection in Multi- and Hyperspectral Data," IEEE Trans. Image Process., vol. 16, no. 2, pp. 463-478, Feb. 2007.

[16] M. J. Canty, A. A. Nielsen, and M. Schmidt, "Automatic radiometric normalization of multitemporal satellite imagery," Remote Sens. Environ., vol. 91, no. 3, pp. 441-451, Jun. 2004.

[17] M. J. Canty and A. A. Nielsen, "Automatic radiometric normalization of multitemporal satellite imagery with the iteratively re-weighted MAD transformation," Remote Sens. Environ., vol. 112, no. 3, pp. 1025-1036, Mar. 2008.

[18] Y. J. Kaufman, "Atmospheric effect on spectral signaturemeasurements and corrections," IEEE Trans. Geosci. Remote Sens., vol. 26, no. 4, pp. 441-450, Jul. 1988.

[19] V. CASELLES and M. J. L. GARCÍA, "An alternative simple approach to estimate atmospheric correction in multitemporal studies," Int. J. Remote Sens., vol. 10, no. 6, pp. 1127-1134, Jun. 1989.

[20] Y. Du, P. M. Teillet, and J. Cihlar, "Radiometric normalization of multitemporal high-resolution satellite images with quality control for land cover change detection," Remote Sens. Environ., vol. 82, no. 1, pp. 123-134, Sep. 2002.

[21] A. Baraldi, "Impact of Radiometric Calibration and Specifications of Spaceborne Optical Imaging Sensors on the Development of Operational Automatic Remote Sensing Image Understanding Systems," IEEE J. Sel. Top. Appl. Earth Obs. Remote Sens., vol. 2, no. 2, pp. 104-134, Jun. 2009.

[22] G. Schaepman-Strub, M. E. Schaepman, T. H. Painter, S. Dangel, and J. V. Martonchik, "Reflectance quantities in optical remote sensing-definitions and case studies," Remote Sens. Environ., vol. 103, no. 1, pp. 27-42, Jul. 2006.

[23] F. G. Hall, D. E. Strebel, J. E. Nickeson, and S. J. Goetz, "Radiometric rectification: Toward a common radiometric response among multidate, multisensor images," Remote Sens. Environ., vol. 35, no. 1, pp. 11-27, Jan. 1991.

[24] J. E. CONEL, "Determination of surface reflectance and estimates of atmospheric optical depth and single scattering albedo from Landsat Thematic Mapper data," Int. J. Remote Sens., vol. 11, no. 5, pp. 783-828, May 1990.

[25] P. R. Coppin and M. E. Bauer, "Processing of multitemporal Landsat TM imagery to optimize extraction of forest cover change features," IEEE Trans. Geosci. Remote Sens., vol. 32, no. 4, pp. 918-927, Jul. 1994.

[26] P. S. Chavez and D. J. Mackinnon, "Automatic Detection of Vegetation Changes in the Southwestern United States Using Remotely Sensed Images," Photogramm. Eng. Remote Sens., vol. 60, no. 5, May 1994.

[27] X. J. Yang and C. P. Lo, "Relative radiometric normalization performance for change detection from multi-date satellite 
images," Photogramm. Eng. Remote Sens., vol. 66, no. 8, pp. 967-980, Aug. 2000.

[28] J. R. Schott, C. Salvaggio, and W. J. Volchok, "Radiometric scene normalization using pseudoinvariant features," Remote Sens. Environ., vol. 26, no. 1, pp. 1-16, Oct. 1988.

[29] G. S. Burns and A. T. Joyce, "Evaluation of Land Cover Change Detection Techniques Using Landsat Mss Data," in Proceeding of the 7th Pecora Symposium, Sioux Falls, SD, USA, 1981, pp. 252-260.

[30] A. Sinch, Change detection in the tropical forest environment of northeastern India using Landsat. In: "Remote Sensing and Tropical Land Management. London: John Wiley and Sons, 1986.

[31] J. Heo and T. W. FitzHugh, "A standardized radiometric normalization method for change detection using remotely sensed imagery," Photogramm. Eng. Remote Sens., vol. 66, no. 2, pp. 173-181, Feb. 2000.

[32] K. Tan, X. Jin, A. Plaza, X. Wang, L. Xiao, and P. Du, "Automatic Change Detection in High-Resolution Remote Sensing Images by Using a Multiple Classifier System and Spectral-Spatial Features," IEEE J. Sel. Top. Appl. Earth Obs. Remote Sens., vol. 9, no. 8, pp. 3439-3451, Aug. 2016.

[33] P. Zhang, M. Gong, L. Su, J. Liu, and Z. Li, "Change detection based on deep feature representation and mapping transformation for multi-spatial-resolution remote sensing images," ISPRS J. Photogramm. Remote Sens., vol. 116, pp. 24-41, Jun. 2016.

[34] D. G. Lowe, "Distinctive Image Features from Scale-Invariant Keypoints," Int. J. Comput. Vis., vol. 60, no. 2, pp. 91-110, Nov. 2004.

[35] H. Zhang, J. Chen, and Z. Mao, "The research on relative radiometric normalization for change detection of multitemporal images," presented at the Image and Signal Processing for Remote Sensing XV, 2009, vol. 7477, p. 747714.

[36] F. Dellinger, J. Delon, Y. Gousseau, J. Michel, and F. Tupin, "Change detection for high resolution satellite images, based on SIFT descriptors and an a contrario approach," in 2014 IEEE Geoscience and Remote Sensing Symposium, 2014, pp. 12811284.

[37] A. Sedaghat, M. Mokhtarzade, and H. Ebadi, "Uniform Robust Scale-Invariant Feature Matching for Optical Remote Sensing Images," IEEE Trans. Geosci. Remote Sens., vol. 49, no. 11, pp. 4516-4527, Nov. 2011.

[38] V. Sadeghi, H. Ebadi, and F. Farnood-Ahmadi, "A new model for automatic normalization of multitemporal satellite images using Artificial Neural Network and mathematical methods," Appl. Math. Model., vol. 37, no. 9, pp. 6437-6445, May 2013.

[39] F. Bovolo and L. Bruzzone, "A Theoretical Framework for Unsupervised Change Detection Based on Change Vector Analysis in the Polar Domain," IEEE Trans. Geosci. Remote Sens., vol. 45, no. 1, pp. 218-236, Jan. 2007.

[40] D. R. Insua and P. Müller, "Feedforward Neural Networks for Nonparametric Regression," in Practical Nonparametric and Semiparametric Bayesian Statistics, Springer, New York, NY, 1998, pp. 181-193.

[41] H. Drucker, C. J. C. Burges, L. Kaufman, A. J. Smola, and V. Vapnik, "Support Vector Regression Machines," in Advances in Neural Information Processing Systems 9, M. C. Mozer, M. I. Jordan, and T. Petsche, Eds. MIT Press, 1997, pp. 155-161.

[42] J. R. Jensen, "Urban/Suburban Land Use Analysis," in Manual of Remote Sensing, 2nd ed., Falls Church, VA: American Society of Photogrammetry, 1983, pp. 1571-1666.

[43] C. D. Elvidge, D. Yuan, R. D. Werackoon, and R. S. Lunetta, "Relative Radiometric Normalization of Landsat Multispectral Scanner (MSS) Data Using an Automated Scattergram Controlled Regression," Photogramm. Eng. Remote Sens., vol. 61, no. 10, pp. 1255-1260, Oct. 1995.
[44] M. Zanetti, F. Bovolo, and L. Bruzzone, "Rayleigh-Rice Mixture Parameter Estimation via EM Algorithm for Change Detection in Multispectral Images," IEEE Trans. Image Process., vol. 24, no. 12, pp. 5004-5016, Dec. 2015.

[45] Y. T. Solano-Correa, F. Bovolo, and L. Bruzzone, "VHR timeseries generation by prediction and fusion of multi-sensor images," in 2015 IEEE International Geoscience and Remote Sensing Symposium (IGARSS), 2015, pp. 3298-3301.

[46] "DigitalGlobe Foundation." [Online]. Available: http://foundation.digitalglobe.com/. [Accessed: 15-Jan-2018].

[47] "DigitalGlobe Atmospheric Compensation." [Online]. Available:

http://explore.digitalglobe.com/AComp.html?utm_source=blog \& utm_medium=website\&utm_campaign=AComp. [Accessed: 15Jan-2018].

[48] F. Pacifici, "Atmospheric compensation in satellite imagery," US9396528 B2, 19-Jul-2016.

[49] C. Paris and L. Bruzzone, "A Three-Dimensional Model-Based Approach to the Estimation of the Tree Top Height by Fusing Low-Density LiDAR Data and Very High Resolution Optical Images," IEEE Trans. Geosci. Remote Sens., vol. 53, no. 1, pp. 467-480, Jan. 2015.

[50] C. A. Laben and B. V. Brower, "Process for enhancing the spatial resolution of multispectral imagery using pansharpening," US6011875A, 04-Jan-2000.

[51] "ENVI - The Leading Geospatial Analytics Software | Harris Geospatial." [Online]. Available: http://www.harrisgeospatial.com/SoftwareTechnology/ENVI.asp x. [Accessed: 15-Jan-2018].

[52] R. M. Haralick, K. Shanmugam, and I. Dinstein, "Textural Features for Image Classification," IEEE Trans. Syst. Man Cybern., vol. SMC-3, no. 6, pp. 610-621, Nov. 1973.

[53] S. E. Grigorescu, N. Petkov, and P. Kruizinga, "Comparison of texture features based on Gabor filters," IEEE Trans. Image Process., vol. 11, no. 10, pp. 1160-1167, Oct. 2002.

[54] S. Kullback and R. A. Leiber, "On Information and Sufficiency," Ann. Math. Stat., vol. 22, no. 1, pp. 79-86, Mar. 1951.

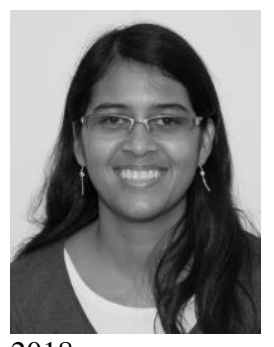

Yady Tatiana Solano-Correa (S'13-M'18) received the Bachelor (B.S.) degree in Physics Engineering (honorable mention) from the University of Cauca, Cauca, Colombia, in 2011; and the Ph.D. degree (magna cum laude) in communication and information technologies from the Department of Information Engineering and Computer Science, University of Trento, Trento, Italy, in 2018.

From 2009 to 2013, she was a Researcher for the research groups: Optics and Laser Group (GOL) and Environmental Studies Group (GEA), University of Cauca. She is currently a Post-Doctoral Researcher with the Remote Sensing for Digital Earth Unit, Fondazione Bruno Kessler, Trento. She is a member of the RSLab, University of Trento. She works, and has worked, within the context of several projects with a focus on analyzing information for climate change and developing advanced change detection techniques for optical satellite time series data, among them: 1) RICCLISAInterinstitutional network of climate change and food security, Colombia. Founded by the Colombian administrative department of Science, Technology and Innovation-COLCIENCIAS; 2) MS-TSAnalysis of MultiSensor VHR image Time Series, in collaboration with Digital Globe Foundation; and 3) SEOM-Scientific Exploitation of Operational Missions-S2-4Sci Land and WaterMultitemporal Analysis. Founded by European Space Agency (ESA). Her research interests include remote sensing environmental 
applications, change detection, both on medium resolution multispectral images (e.g., Landsat and Sentinel-2) and very highresolution (VHR) images, multitemporal analysis of short- and longtime series, multisensor multitemporal image preprocessing and information extraction, pattern recognition and image classification.

Dr. Solano-Correa was a recipient of the Best Student Oral Presentation Award at the MultiTemp 2017 Conference, Bruges, Belgium, in 2017. She is a referee for the IEEE TRANSACTIONS ON GEOSCIENCE AND REMOTE SENSING, the IEEE Journal of Selected Topics in Applied Earth Observations and Remote Sensing, and the IEEE GEOSCIENCE AND REMOTE SENSING LETTERS.

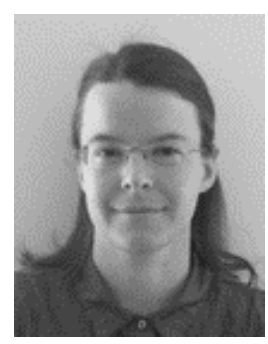

Francesca Bovolo (S'05-M'07-SM'13) received the Laurea (B.S.) degree, the Laurea Specialistica (M.S.) degree (summa cum laude) in telecommunication engineering, and the Ph.D. degree in communication and information technologies from the University of Trento, Trento, Italy, in 2001, 2003, and 2006, respectively.

She was a Research Fellow with the University of Trento, until 2013. She is currently the Founder and the Head of Remote Sensing for Digital Earth Unit, Fondazione Bruno Kessler, Trento, and a member of the Remote Sensing Laboratory, Trento. She is one of the co-investigators of the Radar for Icy Moon Exploration instrument of the European Space Agency Jupiter Icy Moons Explorer. Her research interests include remote-sensing image processing, multitemporal remote sensing image analysis, change detection in multispectral, hyperspectral, and synthetic aperture radar images, and very high-resolution images, time series analysis, content-based time series retrieval, domain adaptation, and Light Detection and Ranging (LiDAR) and radar sounders. She conducts research on these topics within the context of several national and international projects.

Dr. Bovolo is a member of the program and scientific committee of several international conferences and workshops. She was a recipient of the First Place in the Student Prize Paper Competition of the 2006 IEEE International Geoscience and Remote Sensing Symposium (Denver, 2006). She was the Technical Chair of the Sixth International Workshop on the Analysis of Multitemporal RemoteSensing Images (MultiTemp 2011). She has been a Co-Chair of the SPIE International Conference on Signal and Image Processing for Remote Sensing since 2014. She is the Publication Chair of the International Geoscience and Remote Sensing Symposium in 2015. She has been an Associate Editor of the IEEE JOURNAL OF SELECTED TOPICS IN APPLIED EARTH OBSERVATIONS AND REMOTE SENSING since 2011 and the Guest Editor of the Special Issue on Analysis of Multitemporal Remote Sensing Data of the IEEE TRANSACTIONS ON GEOSCIENCE AND REMOTE SENSING. She is a referee for several international journals.

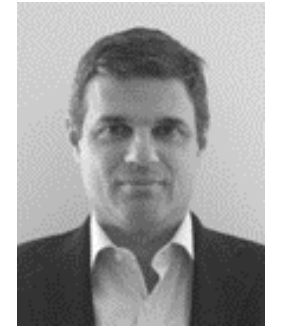

Lorenzo Bruzzone (S'95-M'98-SM'03F'10) received the Laurea (M.S.) degree (summa cum laude) in electronic engineering and the Ph.D. degree in telecommunications from the University of Genoa, Genoa, Italy, in 1993 and 1998, respectively.

$\mathrm{He}$ is currently a Full Professor of telecommunications with the University of Trento, Trento, Italy, where he teaches remote sensing, radar, and digital communications. He is the Founder and the Director of the Remote Sensing Laboratory, Department of Information Engineering and Computer Science, University of Trento. He is the Principal Investigator of many research projects, including the Radar for Icy Moon Exploration instrument in the framework of the Jupiter Icy Moons Explorer mission of the European Space Agency (ESA), and the Science Lead for the HighResolution Land Cover Project in the framework of the Climate Change Initiative of ESA. He has authored or co-authored 236 scientific publications in refereed international journals (172 in IEEE journals), more than 310 papers in conference proceedings, and 21 book chapters. He has edited or co-edited 18 books/conference proceedings and 1 scientific book. His papers are highly cited, as proven from the total number of citations (more than 27000) and the value of the h-index (74) (source: Google Scholar). His research interests include remote sensing, radar and synthetic aperture radar, signal processing, machine learning, and pattern recognition. $\mathrm{He}$ promotes and supervises research on these topics within the frameworks of many national and international projects.

Dr. Bruzzone has been a member of the Administrative Committee of the IEEE Geoscience and Remote Sensing Society (GRSS) since 2009, where he is currently a Vice-President for Professional Activities. He is currently a member of the Permanent Steering Committee of the IEEE International Workshop on the Analysis of Multitemporal Remote-Sensing Images (Multi Temp) series. He was a recipient of the First Place in the Student Prize Paper Competition of the 1998 IEEE International Geoscience and Remote Sensing Symposium (IGARSS), Seattle, in 1998, and many international and national honors and awards, including the recent IEEE GRSS 2015 Outstanding Service Award, the 2017 IEEE IGARSS Symposium Prize Paper Award, and the 2018 IEEE IGARSS Symposium Prize Paper Award. He has been the Chair of the SPIE Conference on Image and Signal Processing for Remote Sensing since 2003. He was a Guest Co-Editor of many special issues of international journals. $\mathrm{He}$ is currently an Associate Editor of the IEEE TRANSACTIONS ON GEOSCIENCE AND REMOTE SENSING. He was the Editorin-Chief of IEEE Geoscience and Remote Sensing Magazine from 2013 to 2017. He is the Co-Founder of the IEEE International Workshop on the Analysis of Multitemporal Remote-Sensing Images (Multi Temp) series. He has been the Founder of IEEE Geoscience and Remote Sensing Magazine. He was invited as a Keynote Speaker in more than 32 international conferences and workshops. He has been a Distinguished Speaker of the IEEE Geoscience and Remote Sensing Society from 2012 to 2016. 